\title{
Ionization Energies and Aqueous Redox Potentials of Organic Molecules: Comparison of DFT, Correlated ab initio Theory and Pair Natural Orbital Approaches
}

\section{Supporting Information}

Miho Isegawa, Frank Neese, Dimitrios A. Pantazis

Max Planck Institute for Chemical Energy Conversion, Stiftrasse 34-38, 45470 Mülheim and der Ruhr, Germany 


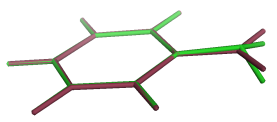

aniline

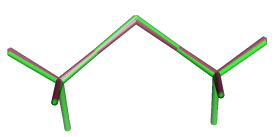

dimethylamine<smiles>CC1CCC2C(C)C1C1C(C)C(C)C(C)C21</smiles>

indole

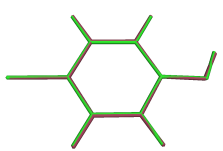

p-chlorophenol

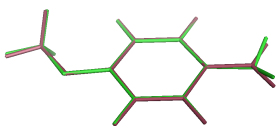

$p$-methoxyaniline

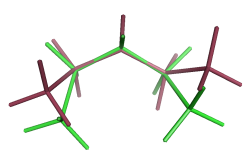

diethylamine

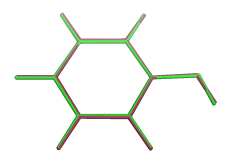

phenol

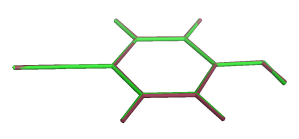

p-cyanophenol

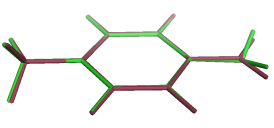

p-methylaniline

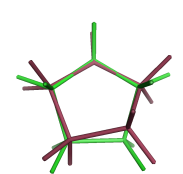

pyrrolidine

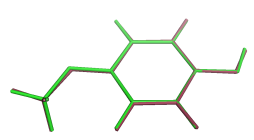

p-methoxyphenol

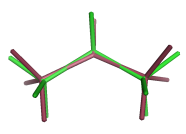

dimethylsulfide

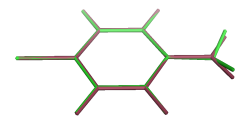

p-chloroaniline

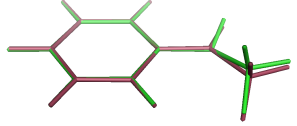

$\mathrm{N}$-methylaniline<smiles>CC1C(C)C(C)C(C)C(C)C1C</smiles>

piperidine

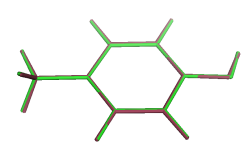

p-methylphenol

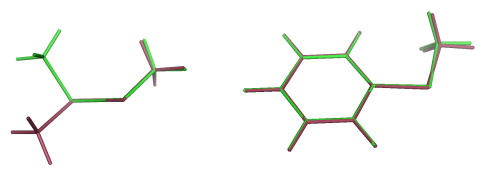

dimethyldisulfide thioanisole

Figure S1. Overlays of gas-phase optimized reduced (green) and oxidized (red) forms. 

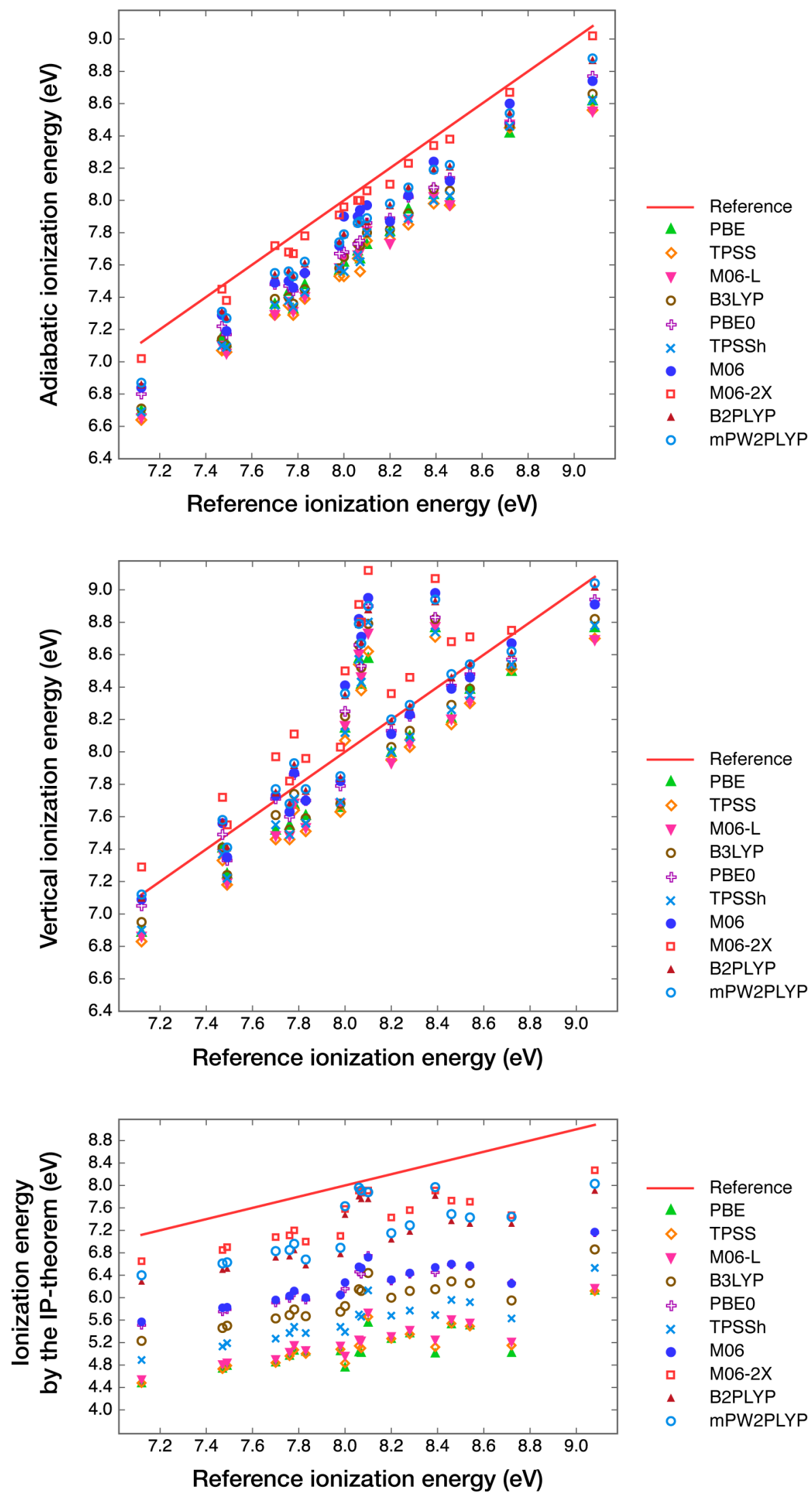

Figure S2. Plots of calculated ionization energies $(\mathrm{eV})$ by ten density functionals compared with reference extrapolated CCSD(T)/cc-pVTZ energies, for adiabatic IEs, vertical IEs, and IEs estimated by HOMO energies ("IP-theorem" values). 
(a) $\mathrm{C}_{6} \mathrm{H}_{5} \mathrm{OH}-\mathrm{H}_{2} \mathrm{O}$

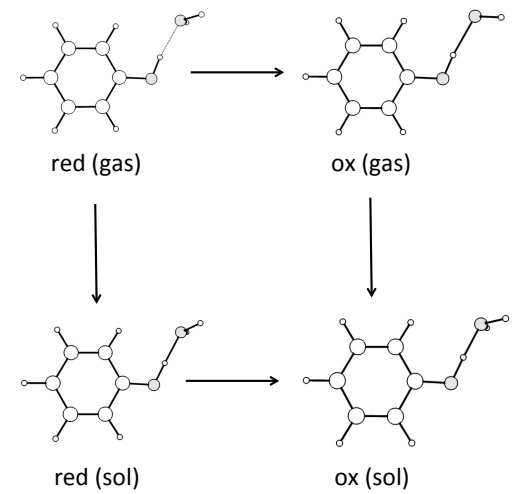

(b) $\mathrm{C}_{6} \mathrm{H}_{5} \mathrm{OH}-\left(\mathrm{H}_{2} \mathrm{O}\right)_{2}$

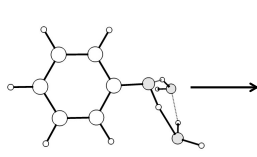

red (gas)

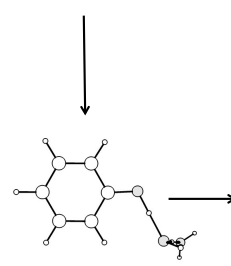

red (sol) (c) $\mathrm{C}_{6} \mathrm{H}_{5} \mathrm{OH}-\left(\mathrm{H}_{2} \mathrm{O}\right)_{3}$

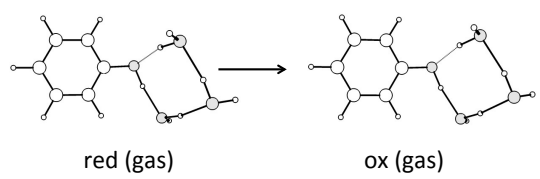

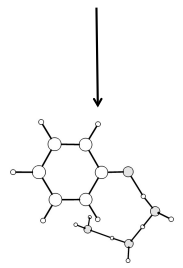

ox (sol)

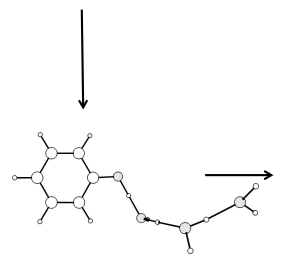

red (sol)

ox (gas)

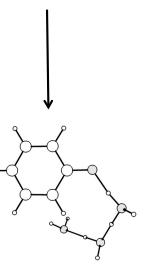

ox (sol)

Figure S3. Optimized structures of three types of phenol-water clusters. 
Table S1. RMSDs of internal coordinates from those of the gas-phase optimized reduced forms for bond lengths ( $\mathrm{A}$ ), bond angles and dihedral angles (degrees).

\begin{tabular}{|c|c|c|c|c|c|c|c|c|c|}
\hline & \multicolumn{3}{|c|}{ Oxidized } & \multicolumn{3}{|c|}{ Reduced in solution } & \multicolumn{3}{|c|}{ Oxidized in solution } \\
\hline & Bonds & Bond angles & Dihedrals & Bonds & Bond angles & Dihedrals & Bonds & Bond angles & Dihedrals \\
\hline aniline & 0.02 & 3.13 & 65.91 & 0.01 & 2.74 & 65.93 & 0.02 & 3.02 & 65.91 \\
\hline$p$-methoxyaniline & 0.14 & 5.17 & 51.44 & 0.14 & 4.44 & 51.45 & 0.14 & 4.92 & 51.44 \\
\hline$p$-methylaniline & 0.05 & 4.03 & 54.39 & 0.04 & 3.62 & 54.16 & 0.05 & 3.92 & 54.33 \\
\hline N-methylaniline & 0.02 & 2.04 & 12.23 & 0.17 & 6.02 & 120.19 & 0.17 & 6.65 & 120.09 \\
\hline dimethylamine & 0.03 & 17.97 & 75.38 & 0.00 & 1.78 & 1.30 & 0.03 & 17.49 & 55.91 \\
\hline diethylamine & 0.01 & 4.07 & 31.41 & 0.01 & 1.35 & 4.65 & 0.01 & 3.46 & 77.72 \\
\hline pyrrolidine & 0.01 & 4.30 & 31.78 & 0.00 & 0.82 & 19.26 & 0.01 & 4.22 & 31.88 \\
\hline piperidine & 0.02 & 2.83 & 21.47 & 0.00 & 0.69 & 1.74 & 0.02 & 2.84 & 36.68 \\
\hline anisole & 0.02 & 1.65 & 0.01 & 0.00 & 0.22 & 0.08 & 0.02 & 1.45 & 0.00 \\
\hline indole & 0.02 & 1.01 & 49.92 & 0.00 & 0.13 & 0.00 & 0.02 & 1.02 & 49.92 \\
\hline$p$-methoxyphenol & 0.03 & 1.74 & 0.73 & 0.00 & 0.29 & 0.75 & 0.02 & 1.41 & 0.74 \\
\hline$p$-methylphenol & 0.02 & 1.45 & 0.79 & 0.00 & 0.25 & 0.05 & 0.02 & 1.33 & 0.73 \\
\hline phenol & 0.02 & 1.61 & 0.00 & 0.33 & 24.86 & 127.27 & 0.33 & 30.54 & 124.55 \\
\hline p-cyanophenol & 0.02 & 1.33 & 48.20 & 0.00 & 0.34 & 8.09 & 0.02 & 1.32 & 44.44 \\
\hline dimethylsulfide & 0.01 & 2.32 & 1.43 & 0.00 & 0.35 & 0.17 & 0.02 & 2.23 & 1.55 \\
\hline dimethyldisulfide & 0.01 & 1.66 & 35.01 & 0.00 & 0.45 & 65.97 & 0.02 & 1.91 & 34.85 \\
\hline thioanisole & 0.02 & 1.24 & 0.09 & 0.00 & 0.23 & 0.08 & 0.02 & 1.17 & 0.13 \\
\hline$p$-chloroaniline & 0.03 & 2.99 & 66.03 & 0.01 & 2.64 & 66.04 & 0.03 & 2.90 & 66.03 \\
\hline$p$-chlorophenol & 0.03 & 1.37 & 0.00 & 0.00 & 0.34 & 0.01 & 0.03 & 1.24 & 0.01 \\
\hline
\end{tabular}




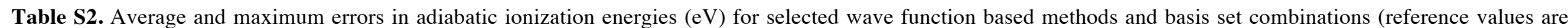
obtained with $\operatorname{CCSD}(\mathrm{T}) / \mathrm{cc}-\mathrm{pVTZ}$ combined with LPNO-CCSD[Q/5] extrapolation of the correlation energy).

\begin{tabular}{|c|c|c|c|c|c|}
\hline Method & Basis set & MUE & MSE & $\mathrm{UE}_{\max }$ & $\mathrm{UE}_{\max }$ species \\
\hline LPNO-CCSD & cc-pVDZ & 0.43 & -0.43 & 0.50 & aniline \\
\hline LPNO-CCSD & cc-pVTZ & 0.21 & -0.20 & 0.63 & chlorophenol \\
\hline LPNO-CCSD & cc-pVQZ & 0.10 & -0.10 & 0.14 & methylaniline \\
\hline LPNO-CCSD & cc-pV(D/T)Z & 0.09 & -0.06 & 0.49 & chlorophenol \\
\hline LPNO-CCSD & cc-pV[T/Q]Z & 0.09 & -0.06 & 0.51 & chlorophenol \\
\hline LPNO-CEPA & cc-pVDZ & 0.47 & -0.47 & 0.58 & cyanophenol \\
\hline LPNO-CEPA & cc-pVTZ & 0.23 & -0.23 & 0.68 & chlorophenol \\
\hline LPNO-CEPA & cc-pVQZ & 0.10 & -0.10 & 0.14 & methylaniline \\
\hline LPNO-CEPA & cc-pV(D/T)Z & 0.11 & -0.08 & 0.54 & chlorophenol \\
\hline LPNO-CEPA & cc-pV[T/Q]Z & 0.12 & -0.08 & 0.55 & chlorophenol \\
\hline LPNO-pCCSD & cc-pVDZ & 0.41 & -0.41 & 0.48 & cyanophenol \\
\hline LPNO-pCCSD & cc-pVTZ & 0.17 & -0.16 & 0.61 & chlorophenol \\
\hline LPNO-pCCSD & cc-pVQZ & 0.06 & -0.06 & 0.10 & aniline \\
\hline LPNO-pCCSD & cc-pV(D/T)Z & 0.05 & -0.02 & 0.46 & chlorophenol \\
\hline LPNO-pCCSD & cc-pV[T/Q]Z & 0.05 & -0.02 & 0.48 & chlorophenol \\
\hline $\mathrm{F} 12-\mathrm{CCSD}(\mathrm{T})$ & cc-pVDZ & 0.13 & -0.13 & 0.17 & chloroaniline \\
\hline CCSD & cc-pVDZ & 0.45 & -0.45 & 0.52 & cyanophenol \\
\hline CCSD & cc-pVTZ & 0.22 & -0.22 & 0.26 & aniline \\
\hline CCSD & cc-pV[D/T]Z & 0.08 & -0.08 & 0.12 & aniline \\
\hline $\operatorname{CCSD}(\mathrm{T})$ & cc-pVDZ & 0.41 & -0.41 & 0.47 & cyanophenol \\
\hline $\operatorname{CCSD}(\mathrm{T})$ & cc-pVTZ & 0.14 & -0.14 & 0.16 & methoxyphenol \\
\hline $\operatorname{CCSD}(\mathrm{T})$ & cc-pV[D/T]Z & 0.02 & 0.02 & 0.05 & thioanisole \\
\hline MP2 & cc-pVDZ & 0.29 & 0.12 & 0.69 & cyanophenol \\
\hline
\end{tabular}




\begin{tabular}{llllll} 
MP2 & cc-pVTZ & 0.45 & 0.43 & 1.08 & cyanophenol \\
MP2 & cc-pVQZ & 0.57 & 0.56 & 1.22 & cyanophenol \\
MP2 & cc-pV(D/T)Z & 0.62 & 0.62 & 1.31 & cyanophenol \\
MP2 & cc-pV[T/Q]Z & 0.65 & 0.65 & 1.39 & aniline \\
RI-MP2 & cc-pVTZ & 0.45 & 0.43 & 1.08 & cyanophenol \\
RI-MP2 & cc-pV(D/T)Z & 0.62 & 0.62 & 1.31 & cyanophenol \\
RI-MP2 & cc-pV(T/Q)Z & 0.65 & 0.65 & 1.39 & aniline \\
SCS-MP2 & cc-pVDZ & 0.23 & 0.03 & 0.56 & cyanophenol \\
SCS-MP2 & cc-pVTZ & 0.35 & 0.34 & 0.95 & cyanophenol \\
SCS-MP2 & cc-pVQZ & 0.46 & 0.46 & 1.09 & cyanophenol \\
SCS-MP2 & cc-pV(D/T)Z & 0.52 & 0.52 & 1.17 & cyanophenol \\
SCS-MP2 & cc-pV[T/Q]Z & 0.54 & 0.54 & 1.19 & cyanophenol \\
OO-RI-MP2 & cc-pVDZ & 0.39 & -0.39 & 0.47 & cyanophenol \\
OO-RI-MP2 & cc-pVTZ & 0.11 & -0.11 & 0.19 & dimethyldisulfide \\
OO-RI-MP2 & cc-pVQZ & 0.04 & -0.01 & 0.10 & dimethyldisulfide \\
OO-RI-MP2 & cc-pV(D/T)Z & 0.06 & 0.06 & 0.13 & phenol \\
OO-RI-MP2 & cc-pV[T/Q]Z & 0.07 & 0.06 & 0.13 & pyrrolidine \\
F12-MP2 & cc-pVTZ-F12 & 0.63 & 0.63 & 1.32 & cyanophenol \\
HF & cc-pVTZ & 1.42 & -1.42 & 1.63 & N_methylaniline \\
\hline
\end{tabular}




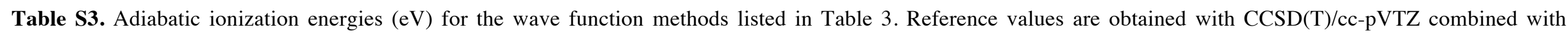
LPNO-CCSD[Q/5] extrapolation of the correlation energy.

\begin{tabular}{|c|c|c|c|c|c|c|c|c|c|c|c|}
\hline & $\operatorname{CCSD}(\mathrm{T})$ & LPNO-pCCSD & OO-MP2 & CCSD & LPNO-CCSD & LPNO-CEPA & $\mathrm{F} 12-\mathrm{CCSD}(\mathrm{T})$ & SCS-MP2 & F12-MP2 & MP2 & $\mathrm{HF}$ \\
\hline & cc-pV[D/T]Z & cc-pV[T/Q]Z & cc-pV[T/Q]Z & cc-pV[D/T]Z & cc-pV[T/Q]Z & cc-pV[T/Q]Z & cc-pVDZ-F12 & cc-pV[T/Q]Z & cc-pVTZ-F12 & cc-pV[T/Q]Z & cc-pVTZ \\
\hline aniline & 7.77 & 7.72 & 7.65 & 7.64 & 7.68 & 7.64 & 7.64 & 8.59 & 8.64 & 9.15 & 6.14 \\
\hline$p$-methoxyaniline & 7.13 & 7.13 & 7.15 & 7.04 & 7.06 & 7.01 & 6.98 & 7.75 & 7.75 & 7.85 & 5.79 \\
\hline$p$-methylaniline & 7.51 & 7.46 & 7.58 & 7.38 & 7.40 & 7.36 & 7.36 & 8.18 & 8.32 & 8.31 & 5.95 \\
\hline N-methylaniline & 7.49 & 7.44 & 7.56 & 7.36 & 7.40 & 7.36 & 7.34 & 8.34 & 8.48 & 8.47 & 5.84 \\
\hline dimethylamine & 8.41 & 8.38 & 8.50 & 8.31 & 8.32 & 8.31 & 8.31 & 8.58 & 8.62 & 8.61 & 6.93 \\
\hline diethylamine & 8.02 & 8.00 & 8.10 & 7.93 & 7.95 & 7.93 & 7.90 & 8.20 & 8.25 & 8.24 & 6.52 \\
\hline pyrrolidine & 8.09 & 8.06 & 8.19 & 7.99 & 8.00 & 7.99 & 7.97 & 8.28 & 8.32 & 8.31 & 6.60 \\
\hline piperidine & 8.09 & 8.10 & 8.11 & 8.02 & 8.07 & 8.03 & 7.96 & 8.32 & 8.35 & 8.34 & 6.75 \\
\hline anisole & 8.30 & 8.28 & 8.38 & 8.18 & 8.22 & 8.18 & 8.16 & 8.88 & 9.04 & 9.03 & 6.76 \\
\hline indole & 7.84 & 7.80 & 7.89 & 7.73 & 7.77 & 8.06 & 7.69 & 8.78 & 8.96 & 8.95 & 6.21 \\
\hline$p$-methoxyphenol & 7.71 & 7.72 & 7.75 & 7.63 & 7.66 & 7.61 & 7.56 & 7.97 & 8.08 & 8.08 & 6.41 \\
\hline$p$-methylphenol & 8.22 & 8.20 & 8.31 & 8.10 & 8.12 & 8.10 & 8.07 & 8.65 & 8.79 & 8.78 & 6.76 \\
\hline phenol & 8.56 & 8.53 & 8.66 & 8.43 & 8.46 & 8.44 & 8.42 & 9.09 & 9.25 & 9.24 & 7.00 \\
\hline$p$-cyanophenol & 9.11 & 9.07 & 9.14 & 9.00 & 9.04 & 8.97 & 8.93 & 10.27 & 10.40 & 10.40 & 7.53 \\
\hline dimethylsulfide & 8.76 & 8.75 & 8.77 & 8.68 & 8.69 & 8.69 & 8.60 & 8.81 & 8.83 & 8.82 & 7.52 \\
\hline dimethyldisulfide & 8.13 & 8.14 & 8.06 & 8.11 & 8.15 & 8.13 & 7.95 & 8.18 & 8.15 & 8.14 & 7.25 \\
\hline thioanisole & 8.03 & 7.98 & 8.00 & 7.94 & 7.95 & 7.91 & 7.83 & 8.80 & 8.89 & 8.89 & 6.61 \\
\hline$p$-chloroaniline & 7.79 & 7.98 & 7.81 & 7.67 & 7.95 & 7.91 & 7.61 & 8.56 & 8.68 & 8.68 & 6.31 \\
\hline$p$-chlorophenol & 8.48 & 7.98 & 8.51 & 8.39 & 7.95 & 7.91 & 8.30 & 9.01 & 9.13 & 9.13 & 7.15 \\
\hline MUE & 0.02 & 0.05 & 0.07 & 0.08 & 0.09 & 0.12 & 0.13 & 0.54 & 0.63 & 0.65 & 1.42 \\
\hline MSE & 0.02 & -0.02 & 0.06 & -0.08 & -0.06 & -0.08 & -0.13 & 0.54 & 0.63 & 0.65 & -1.42 \\
\hline
\end{tabular}




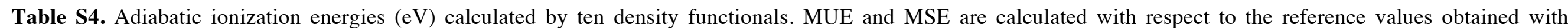
CCSD(T)/cc-pVTZ combined with LPNO-CCSD[Q/5] extrapolation.

\begin{tabular}{|c|c|c|c|c|c|c|c|c|c|c|c|c|c|}
\hline & PBE & TPSS & M06-L & B3LYP & PBE0 & TPSSh & M06 & M06- & B2PLYP & mPW2PLYP & MUE & MSE & REF \\
\hline aniline & 7.44 & 7.35 & 7.37 & 7.40 & 7.47 & 7.38 & 7.50 & 7.68 & 7.56 & 7.56 & 0.29 & -0.29 & 7.76 \\
\hline$p$-methoxyaniline & 6.70 & 6.64 & 6.65 & 6.71 & 6.80 & 6.69 & 6.84 & 7.02 & 6.86 & 6.87 & 0.34 & -0.34 & 7.12 \\
\hline$p$-methylaniline & 7.12 & 7.06 & 7.05 & 7.10 & 7.17 & 7.09 & 7.19 & 7.38 & 7.27 & 7.27 & 0.32 & -0.32 & 7.49 \\
\hline $\mathrm{N}$-methylaniline & 7.16 & 7.07 & 7.10 & 7.14 & 7.22 & 7.10 & 7.29 & 7.45 & 7.31 & 7.31 & 0.25 & -0.25 & 7.47 \\
\hline dimethylamine & 8.04 & 7.98 & 8.02 & 8.07 & 8.08 & 8.00 & 8.24 & 8.34 & 8.19 & 8.19 & 0.28 & -0.28 & 8.39 \\
\hline diethylamine & 7.62 & 7.53 & 7.64 & 7.65 & 7.68 & 7.56 & 7.90 & 7.96 & 7.79 & 7.79 & 0.29 & -0.29 & 8.00 \\
\hline pyrrolidine & 7.69 & 7.64 & 7.66 & 7.73 & 7.73 & 7.66 & 7.90 & 8.00 & 7.86 & 7.86 & 0.29 & -0.29 & 8.06 \\
\hline piperidine & 7.64 & 7.56 & 7.68 & 7.72 & 7.75 & 7.62 & 7.94 & 8.00 & 7.86 & 7.87 & 0.31 & -0.31 & 8.07 \\
\hline anisole & 7.95 & 7.85 & 7.88 & 7.92 & 8.02 & 7.89 & 8.03 & 8.23 & 8.08 & 8.08 & 0.29 & -0.29 & 8.28 \\
\hline indole & 7.48 & 7.39 & 7.40 & 7.45 & 7.55 & 7.43 & 7.55 & 7.78 & 7.61 & 7.62 & 0.30 & -0.30 & 7.83 \\
\hline p-methoxyphenol & 7.36 & 7.29 & 7.29 & 7.39 & 7.48 & 7.35 & 7.49 & 7.72 & 7.54 & 7.55 & 0.26 & -0.25 & 7.70 \\
\hline$p$-methylphenol & 7.81 & 7.76 & 7.73 & 7.82 & 7.89 & 7.80 & 7.87 & 8.10 & 7.97 & 7.98 & 0.33 & -0.33 & 8.20 \\
\hline phenol & 9.27 & 9.20 & 9.22 & 9.22 & 9.32 & 9.23 & 9.22 & 9.48 & 9.42 & 9.42 & 0.76 & 0.76 & 8.54 \\
\hline p-cyanophenol & 8.62 & 8.56 & 8.55 & 8.66 & 8.77 & 8.62 & 8.74 & 9.02 & 8.87 & 8.88 & 0.35 & -0.35 & 9.08 \\
\hline dimethylsulfide & 8.42 & 8.45 & 8.47 & 8.46 & 8.48 & 8.46 & 8.60 & 8.67 & 8.54 & 8.54 & 0.21 & -0.21 & 8.72 \\
\hline dimethyldisulfide & 7.73 & 7.75 & 7.81 & 7.80 & 7.86 & 7.80 & 7.97 & 8.06 & 7.87 & 7.89 & 0.25 & -0.25 & 8.10 \\
\hline thioanisole & 7.57 & 7.53 & 7.58 & 7.58 & 7.67 & 7.58 & 7.72 & 7.91 & 7.74 & 7.74 & 0.32 & -0.32 & 7.98 \\
\hline$p$-chloroaniline & 7.33 & 7.29 & 7.31 & 7.36 & 7.44 & 7.34 & 7.46 & 7.67 & 7.53 & 7.53 & 0.35 & -0.35 & 7.78 \\
\hline$p$-chlorophenol & 8.00 & 7.97 & 7.97 & 8.06 & 8.14 & 8.03 & 8.12 & 8.38 & 8.21 & 8.22 & 0.35 & -0.35 & 8.46 \\
\hline MUE & 0.40 & 0.45 & 0.42 & 0.38 & 0.32 & 0.41 & 0.25 & 0.11 & 0.25 & 0.24 & & & \\
\hline MSE & -0.32 & -0.38 & -0.35 & -0.30 & -0.24 & -0.34 & -0.18 & -0.01 & -0.16 & -0.15 & & & \\
\hline
\end{tabular}


Table S5. Errors in ionization energies (eV) for Hartree-Fock, MP2, and OO-MP2, and singles amplitudes obtained from the latter.

\begin{tabular}{lcccc}
\hline & HF error & MP2 error & OO-MP2 error & singles amplitudes \\
\hline aniline & -1.62 & 0.67 & -0.08 & 0.08 \\
$p$-methoxyaniline & -1.33 & 0.43 & -0.17 & 0.12 \\
$p$-methylaniline & -1.54 & 0.63 & -0.09 & 0.09 \\
N-methylaniline & -1.63 & 0.80 & -0.09 & 0.09 \\
dimethylamine & -1.46 & 0.04 & -0.07 & 0.05 \\
diethylamine & -1.48 & 0.07 & -0.07 & 0.07 \\
pyrrolidine & -1.46 & 0.08 & -0.06 & 0.06 \\
piperidine & -1.32 & 0.11 & -0.11 & 0.09 \\
anisole & -1.52 & 0.57 & -0.07 & 0.12 \\
indole & -1.62 & 0.92 & -0.11 & 0.09 \\
$p$-methoxyphenol & -1.29 & 0.18 & -0.15 & 0.13 \\
$p$-methylphenol & -1.44 & 0.41 & -0.06 & 0.11 \\
phenol & -1.54 & 0.51 & -0.06 & 0.10 \\
$p$-cyanophenol & -1.55 & 1.08 & -0.13 & 0.14 \\
dimethylsulfide & -1.20 & -0.05 & -0.10 & 0.03 \\
dimethyldisulfide & -0.85 & -0.11 & -0.19 & 0.03 \\
thioanisole & -1.37 & 0.72 & -0.13 & 0.06 \\
$p$-chloroaniline & -1.47 & 0.69 & -0.15 & 0.10 \\
$p$-chlorophenol & -1.31 & 0.47 & -0.13 & 0.12 \\
\hline
\end{tabular}


Table S6. Spin-squared expectation values for the oxidized form in the gas phase calculated by ten density functionals.

\begin{tabular}{|c|c|c|c|c|c|c|c|c|c|c|c|c|c|}
\hline & PBE & TPSS & M06-L & B3LYP & PBE0 & TPSSh & M06 & M06-2X & B2PLYP & mPW2PLYP & MUE & MSE & REF \\
\hline aniline & 0.76 & 0.76 & 0.77 & 0.77 & 0.78 & 0.77 & 0.77 & 0.78 & 0.83 & 0.84 & 0.03 & 0.03 & 0.75 \\
\hline$p$-methoxyaniline & 0.75 & 0.75 & 0.76 & 0.76 & 0.76 & 0.76 & 0.76 & 0.77 & 0.78 & 0.78 & 0.01 & 0.01 & 0.75 \\
\hline$p$-methylaniline & 0.76 & 0.76 & 0.76 & 0.76 & 0.77 & 0.76 & 0.77 & 0.77 & 0.81 & 0.81 & 0.02 & 0.02 & 0.75 \\
\hline N-methylaniline & 0.76 & 0.76 & 0.77 & 0.77 & 0.78 & 0.77 & 0.78 & 0.78 & 0.85 & 0.85 & 0.04 & 0.04 & 0.75 \\
\hline dimethylamine & 0.75 & 0.75 & 0.76 & 0.75 & 0.76 & 0.76 & 0.76 & 0.76 & 0.76 & 0.76 & 0.01 & 0.01 & 0.75 \\
\hline diethylamine & 0.75 & 0.75 & 0.76 & 0.75 & 0.76 & 0.76 & 0.76 & 0.76 & 0.76 & 0.76 & 0.01 & 0.01 & 0.75 \\
\hline pyrrolidine & 0.75 & 0.75 & 0.76 & 0.75 & 0.76 & 0.76 & 0.76 & 0.76 & 0.76 & 0.76 & 0.01 & 0.01 & 0.75 \\
\hline piperidine & 0.75 & 0.75 & 0.76 & 0.75 & 0.76 & 0.75 & 0.76 & 0.76 & 0.76 & 0.76 & 0.01 & 0.01 & 0.75 \\
\hline anisole & 0.76 & 0.76 & 0.76 & 0.77 & 0.77 & 0.77 & 0.77 & 0.77 & 0.81 & 0.81 & 0.03 & 0.03 & 0.75 \\
\hline indole & 0.76 & 0.76 & 0.76 & 0.76 & 0.77 & 0.76 & 0.77 & 0.77 & 0.81 & 0.81 & 0.02 & 0.02 & 0.75 \\
\hline$p$-methoxyphenol & 0.75 & 0.75 & 0.75 & 0.76 & 0.76 & 0.76 & 0.76 & 0.77 & 0.78 & 0.78 & 0.01 & 0.01 & 0.75 \\
\hline$p$-methylphenol & 0.75 & 0.76 & 0.76 & 0.76 & 0.76 & 0.76 & 0.76 & 0.77 & 0.78 & 0.78 & 0.01 & 0.01 & 0.75 \\
\hline phenol & 0.76 & 0.76 & 0.76 & 0.76 & 0.77 & 0.77 & 0.77 & 0.77 & 0.80 & 0.81 & 0.02 & 0.02 & 0.75 \\
\hline p-cyanophenol & 0.76 & 0.76 & 0.76 & 0.77 & 0.78 & 0.77 & 0.77 & 0.78 & 0.83 & 0.84 & 0.03 & 0.03 & 0.75 \\
\hline dimethylsulfide & 0.75 & 0.76 & 0.76 & 0.75 & 0.76 & 0.76 & 0.75 & 0.75 & 0.76 & 0.76 & 0.01 & 0.01 & 0.75 \\
\hline dimethyldisulfide & 0.75 & 0.75 & 0.75 & 0.76 & 0.76 & 0.76 & 0.75 & 0.75 & 0.76 & 0.76 & 0.01 & 0.01 & 0.75 \\
\hline thioanisole & 0.76 & 0.76 & 0.76 & 0.76 & 0.77 & 0.76 & 0.77 & 0.77 & 0.81 & 0.82 & 0.02 & 0.02 & 0.75 \\
\hline$p$-chloroaniline & 0.75 & 0.76 & 0.76 & 0.76 & 0.77 & 0.76 & 0.77 & 0.77 & 0.81 & 0.82 & 0.02 & 0.02 & 0.75 \\
\hline$p$-chlorophenol & 0.75 & 0.75 & 0.76 & 0.76 & 0.76 & 0.76 & 0.76 & 0.77 & 0.78 & 0.78 & 0.01 & 0.01 & 0.75 \\
\hline MUE & 0.00 & 0.01 & 0.01 & 0.01 & 0.02 & 0.01 & 0.01 & 0.02 & 0.04 & 0.04 & & & \\
\hline MSE & 0.00 & 0.01 & 0.01 & 0.01 & 0.02 & 0.01 & 0.01 & 0.02 & 0.04 & 0.04 & & & \\
\hline
\end{tabular}


Table S7. Spin-squared expectation values for the oxidized forms in aqueous solution calculated by ten density functionals.

\begin{tabular}{|c|c|c|c|c|c|c|c|c|c|c|c|c|c|}
\hline & PBE & TPSS & M06-L & B3LYP & PBE0 & TPSSh & M06 & M06-2X & B2PLYP & mPW2PLYP & MUE & MSE & REF \\
\hline aniline & 0.76 & 0.77 & 0.77 & 0.77 & 0.79 & 0.78 & 0.78 & 0.78 & 0.85 & 0.86 & 0.04 & 0.04 & 0.75 \\
\hline$p$-methoxyaniline & 0.75 & 0.76 & 0.76 & 0.76 & 0.77 & 0.76 & 0.76 & 0.77 & 0.80 & 0.80 & 0.02 & 0.02 & 0.75 \\
\hline$p$-methylaniline & 0.76 & 0.76 & 0.77 & 0.77 & 0.78 & 0.77 & 0.77 & 0.77 & 0.83 & 0.84 & 0.03 & 0.03 & 0.75 \\
\hline $\mathrm{N}$-methylaniline & 0.76 & 0.77 & 0.77 & 0.78 & 0.79 & 0.78 & 0.78 & 0.78 & 0.86 & 0.87 & 0.04 & 0.04 & 0.75 \\
\hline dimethylamine & 0.75 & 0.75 & 0.76 & 0.75 & 0.76 & 0.76 & 0.76 & 0.76 & 0.76 & 0.76 & 0.01 & 0.01 & 0.75 \\
\hline diethylamine & 0.75 & 0.75 & 0.76 & 0.75 & 0.76 & 0.76 & 0.76 & 0.76 & 0.76 & 0.76 & 0.01 & 0.01 & 0.75 \\
\hline pyrrolidine & 0.75 & 0.75 & 0.76 & 0.75 & 0.76 & 0.76 & 0.76 & 0.76 & 0.76 & 0.76 & 0.01 & 0.01 & 0.75 \\
\hline piperidine & 0.75 & 0.75 & 0.76 & 0.75 & 0.76 & 0.76 & 0.76 & 0.76 & 0.76 & 0.76 & 0.01 & 0.01 & 0.75 \\
\hline anisole & 0.76 & 0.76 & 0.76 & 0.77 & 0.77 & 0.77 & 0.77 & 0.77 & 0.81 & 0.81 & 0.03 & 0.03 & 0.75 \\
\hline indole & 0.76 & 0.76 & 0.76 & 0.76 & 0.77 & 0.76 & 0.77 & 0.77 & 0.82 & 0.82 & 0.03 & 0.03 & 0.75 \\
\hline$p$-methoxyphenol & 0.75 & 0.75 & 0.75 & 0.76 & 0.76 & 0.76 & 0.76 & 0.77 & 0.77 & 0.77 & 0.01 & 0.01 & 0.75 \\
\hline$p$-methylphenol & 0.75 & 0.76 & 0.76 & 0.76 & 0.76 & 0.76 & 0.76 & 0.77 & 0.78 & 0.78 & 0.01 & 0.01 & 0.75 \\
\hline phenol & 0.76 & 0.76 & 0.76 & 0.77 & 0.77 & 0.77 & 0.77 & 0.77 & 0.81 & 0.81 & 0.03 & 0.03 & 0.75 \\
\hline$p$-cyanophenol & 0.76 & 0.76 & 0.77 & 0.77 & 0.78 & 0.77 & 0.78 & 0.78 & 0.84 & 0.85 & 0.04 & 0.04 & 0.75 \\
\hline dimethylsulfide & 0.75 & 0.76 & 0.76 & 0.75 & 0.76 & 0.76 & 0.75 & 0.75 & 0.76 & 0.76 & 0.01 & 0.01 & 0.75 \\
\hline dimethyldisulfide & 0.75 & 0.75 & 0.75 & 0.76 & 0.76 & 0.76 & 0.75 & 0.75 & 0.76 & 0.76 & 0.01 & 0.01 & 0.75 \\
\hline thioanisole & 0.76 & 0.76 & 0.76 & 0.77 & 0.77 & 0.77 & 0.77 & 0.77 & 0.82 & 0.82 & 0.03 & 0.03 & 0.75 \\
\hline$p$-chloroaniline & 0.76 & 0.76 & 0.77 & 0.77 & 0.78 & 0.77 & 0.77 & 0.78 & 0.85 & 0.85 & 0.04 & 0.04 & 0.75 \\
\hline$p$-chlorophenol & 0.75 & 0.76 & 0.76 & 0.76 & 0.76 & 0.76 & 0.76 & 0.77 & 0.79 & 0.79 & 0.02 & 0.02 & 0.75 \\
\hline MUE & 0.00 & 0.01 & 0.01 & 0.01 & 0.02 & 0.02 & 0.02 & 0.02 & 0.05 & 0.05 & & & \\
\hline MSE & 0.00 & 0.01 & 0.01 & 0.01 & 0.02 & 0.02 & 0.02 & 0.02 & 0.05 & 0.05 & & & \\
\hline
\end{tabular}


Table S8. Contributions (in eV) of zero point vibrational energy, thermal energy, and entropy.

\begin{tabular}{lccc}
\hline & ZPVE & Thermal & Entropy \\
\hline aniline & 0.02 & 0.00 & -0.01 \\
$p$-methoxyaniline & 0.03 & 0.00 & -0.01 \\
$p$-methylaniline & -0.12 & -0.05 & 0.09 \\
N-methylaniline & 0.01 & 0.01 & -0.04 \\
dimethylamine & -0.06 & 0.00 & -0.03 \\
diethylamine & -0.03 & -0.01 & 0.01 \\
pyrrolidine & -0.05 & 0.00 & -0.01 \\
piperidine & -0.02 & 0.01 & -0.03 \\
anisole & -0.01 & 0.01 & -0.04 \\
indole & 0.02 & -0.01 & -0.01 \\
$p$-methoxyphenol & 0.03 & 0.00 & -0.01 \\
$p$-methylphenol & 0.00 & 0.00 & -0.02 \\
phenol & 0.01 & 0.00 & -0.02 \\
$p$-cyanophenol & -0.01 & 0.00 & -0.03 \\
dimethylsulfide & -0.04 & 0.01 & -0.06 \\
dimethyldisulfide & 0.00 & 0.01 & -0.04 \\
thioanisole & 0.00 & 0.00 & -0.02 \\
$p$-chloroaniline & 0.02 & 0.00 & -0.01 \\
$p$-chlorophenol & 0.02 & 0.00 & -0.02 \\
\hline
\end{tabular}


Table S9. Vertical ionization energies $(\mathrm{eV})$ calculated by ten density functionals. The zero point vibrational energy correction is not considered.

\begin{tabular}{|c|c|c|c|c|c|c|c|c|c|c|c|c|c|}
\hline & $\mathrm{PBE}$ & TPSS & M06-L & B3LYP & PBE0 & TPSSh & M06 & M06-2X & B2PLYP & mPW2PLYP & MUE & MSE & REF \\
\hline aniline & 7.55 & 7.46 & 7.48 & 7.52 & 7.60 & 7.49 & 7.63 & 7.82 & 7.68 & 7.68 & 0.18 & -0.17 & 7.76 \\
\hline$p$-methoxyaniline & 6.89 & 6.83 & 6.86 & 6.95 & 7.05 & 6.90 & 7.09 & 7.29 & 7.10 & 7.12 & 0.15 & -0.11 & 7.12 \\
\hline$p$-methylaniline & 7.25 & 7.18 & 7.19 & 7.24 & 7.33 & 7.22 & 7.35 & 7.55 & 7.41 & 7.41 & 0.19 & -0.18 & 7.49 \\
\hline N-methylaniline & 7.41 & 7.33 & 7.37 & 7.41 & 7.49 & 7.37 & 7.56 & 7.72 & 7.57 & 7.58 & 0.10 & 0.01 & 7.47 \\
\hline dimethylamine & 8.77 & 8.71 & 8.77 & 8.82 & 8.83 & 8.74 & 8.98 & 9.07 & 8.93 & 8.94 & 0.47 & 0.47 & 8.39 \\
\hline diethylamine & 8.15 & 8.07 & 8.16 & 8.22 & 8.25 & 8.12 & 8.41 & 8.50 & 8.35 & 8.36 & 0.26 & 0.26 & 8.00 \\
\hline pyrrolidine & 8.58 & 8.54 & 8.60 & 8.66 & 8.66 & 8.57 & 8.82 & 8.91 & 8.79 & 8.79 & 0.63 & 0.63 & 8.06 \\
\hline piperidine & 8.42 & 8.38 & 8.46 & 8.52 & 8.53 & 8.43 & 8.71 & 8.80 & 8.67 & 8.67 & 0.49 & 0.49 & 8.07 \\
\hline anisole & 8.10 & 8.03 & 8.05 & 8.13 & 8.22 & 8.09 & 8.23 & 8.46 & 8.28 & 8.29 & 0.13 & -0.09 & 8.28 \\
\hline indole & 7.61 & 7.51 & 7.53 & 7.59 & 7.70 & 7.56 & 7.70 & 7.96 & 7.76 & 7.77 & 0.19 & -0.16 & 7.83 \\
\hline$p$-methoxyphenol & 7.52 & 7.46 & 7.48 & 7.61 & 7.71 & 7.55 & 7.73 & 7.97 & 7.75 & 7.77 & 0.13 & -0.05 & 7.70 \\
\hline$p$-methylphenol & 8.00 & 7.95 & 7.93 & 8.03 & 8.13 & 8.00 & 8.11 & 8.36 & 8.19 & 8.20 & 0.14 & -0.11 & 8.20 \\
\hline phenol & 8.39 & 8.30 & 8.31 & 8.39 & 8.48 & 8.35 & 8.46 & 8.71 & 8.54 & 8.54 & 0.13 & -0.09 & 8.54 \\
\hline p-cyanophenol & 8.77 & 8.70 & 8.69 & 8.82 & 8.94 & 8.78 & 8.91 & 9.21 & 9.02 & 9.04 & 0.22 & -0.19 & 9.08 \\
\hline dimethylsulfide & 8.50 & 8.51 & 8.55 & 8.53 & 8.57 & 8.54 & 8.67 & 8.75 & 8.61 & 8.62 & 0.14 & -0.14 & 8.72 \\
\hline dimethyldisulfide & 8.58 & 8.62 & 8.73 & 8.79 & 8.90 & 8.80 & 8.95 & 9.12 & 8.88 & 8.90 & 0.73 & 0.73 & 8.10 \\
\hline thioanisole & 7.66 & 7.63 & 7.68 & 7.68 & 7.79 & 7.69 & 7.82 & 8.03 & 7.84 & 7.85 & 0.22 & -0.21 & 7.98 \\
\hline$p$-chloroaniline & 7.68 & 7.64 & 7.68 & 7.74 & 7.86 & 7.71 & 7.87 & 8.11 & 7.92 & 7.93 & 0.12 & 0.03 & 7.78 \\
\hline$p$-chlorophenol & 8.21 & 8.17 & 8.20 & 8.29 & 8.41 & 8.26 & 8.39 & 8.68 & 8.46 & 8.48 & 0.15 & -0.11 & 8.46 \\
\hline MUE & 0.25 & 0.28 & 0.29 & 0.26 & 0.21 & 0.26 & 0.24 & 0.32 & 0.20 & 0.20 & & & \\
\hline MSE & -0.05 & -0.11 & -0.07 & 0.00 & 0.07 & -0.05 & 0.12 & 0.32 & 0.14 & 0.15 & & & \\
\hline
\end{tabular}


Table S10. Ionization energies (eV) estimated by Koopmans' theorem for ten density functionals.

\begin{tabular}{|c|c|c|c|c|c|c|c|c|c|c|c|c|c|}
\hline & PBE & TPSS & M06-L & B3LYP & PBE0 & TPSSh & M06 & M06-2X & B2PLYP & mPW2PLYP & MUE & MSE & REF \\
\hline aniline & 4.97 & 4.96 & 5.03 & 5.69 & 5.99 & 5.37 & 6.03 & 7.11 & 6.74 & 6.85 & 1.89 & -1.89 & 7.76 \\
\hline$p$-methoxyaniline & 4.48 & 4.48 & 4.54 & 5.23 & 5.51 & 4.89 & 5.57 & 6.65 & 6.29 & 6.40 & 1.72 & -1.72 & 7.12 \\
\hline$p$-methylaniline & 4.79 & 4.79 & 4.84 & 5.50 & 5.79 & 5.19 & 5.83 & 6.90 & 6.52 & 6.63 & 1.81 & -1.81 & 7.49 \\
\hline N-methylaniline & 4.74 & 4.73 & 4.81 & 5.46 & 5.75 & 5.13 & 5.82 & 6.85 & 6.50 & 6.61 & 1.83 & -1.83 & 7.47 \\
\hline dimethylamine & 5.01 & 5.12 & 5.25 & 6.15 & 6.45 & 5.69 & 6.54 & 7.91 & 7.82 & 7.97 & 2.00 & -2.00 & 8.39 \\
\hline diethylamine & 4.76 & 4.83 & 4.96 & 5.85 & 6.16 & 5.39 & 6.27 & 7.58 & 7.48 & 7.63 & 1.91 & -1.91 & 8.00 \\
\hline pyrrolidine & 5.03 & 5.14 & 5.25 & 6.15 & 6.46 & 5.70 & 6.55 & 7.91 & 7.81 & 7.96 & 1.66 & -1.66 & 8.06 \\
\hline piperidine & 5.02 & 5.10 & 5.23 & 6.12 & 6.42 & 5.66 & 6.53 & 7.87 & 7.76 & 7.91 & 1.71 & -1.71 & 8.07 \\
\hline anisole & 5.37 & 5.35 & 5.42 & 6.12 & 6.42 & 5.77 & 6.44 & 7.56 & 7.18 & 7.29 & 1.99 & -1.99 & 8.28 \\
\hline indole & 5.04 & 5.00 & 5.06 & 5.67 & 5.96 & 5.37 & 6.00 & 7.00 & 6.58 & 6.68 & 1.99 & -1.99 & 7.83 \\
\hline$p$-methoxyphenol & 4.85 & 4.84 & 4.90 & 5.63 & 5.91 & 5.27 & 5.96 & 7.08 & 6.72 & 6.83 & 1.90 & -1.90 & 7.70 \\
\hline$p$-methylphenol & 5.27 & 5.27 & 5.31 & 6.00 & 6.30 & 5.68 & 6.32 & 7.43 & 7.04 & 7.15 & 2.02 & -2.02 & 8.20 \\
\hline phenol & 5.51 & 5.49 & 5.55 & 6.26 & 6.56 & 5.92 & 6.57 & 7.71 & 7.32 & 7.43 & 2.11 & -2.11 & 8.54 \\
\hline$p$-cyanophenol & 6.13 & 6.12 & 6.17 & 6.86 & 7.16 & 6.53 & 7.17 & 8.27 & 7.91 & 8.03 & 2.04 & -2.04 & 9.08 \\
\hline dimethylsulfide & 5.02 & 5.15 & 5.21 & 5.95 & 6.26 & 5.63 & 6.25 & 7.47 & 7.32 & 7.44 & 2.55 & -2.55 & 8.72 \\
\hline dimethyldisulfide & 5.56 & 5.66 & 5.73 & 6.44 & 6.75 & 6.13 & 6.72 & 7.91 & 7.76 & 7.88 & 1.45 & -1.45 & 8.10 \\
\hline thioanisole & 5.05 & 5.08 & 5.14 & 5.75 & 6.05 & 5.48 & 6.05 & 7.10 & 6.78 & 6.89 & 2.04 & -2.04 & 7.98 \\
\hline$p$-chloroaniline & 5.06 & 5.07 & 5.15 & 5.79 & 6.09 & 5.48 & 6.12 & 7.20 & 6.85 & 6.96 & 1.80 & -1.80 & 7.78 \\
\hline$p$-chlorophenol & 5.53 & 5.54 & 5.61 & 6.29 & 6.59 & 5.96 & 6.60 & 7.73 & 7.37 & 7.49 & 1.99 & -1.99 & 8.46 \\
\hline MUE & 2.94 & 2.91 & 2.84 & 2.11 & 1.81 & 2.46 & 1.77 & 0.62 & 0.91 & 0.79 & & & \\
\hline MSE & -2.94 & -2.91 & -2.84 & -2.11 & -1.81 & -2.46 & -1.77 & -0.62 & -0.91 & -0.79 & & & \\
\hline
\end{tabular}




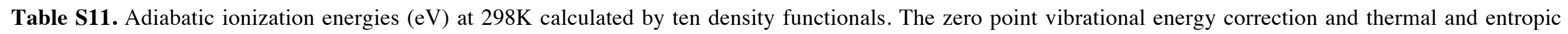
corrections are obtained with TPSS.

\begin{tabular}{|c|c|c|c|c|c|c|c|c|c|c|}
\hline & PBE & TPSS & M06-L & B3LYP & PBE0 & TPSSh & M06 & M06-2X & B2PLYP & mPW2PLYP \\
\hline aniline & 7.45 & 7.36 & 7.38 & 7.41 & 7.48 & 7.39 & 7.51 & 7.69 & 7.57 & 7.57 \\
\hline$p$-methoxyaniline & 6.72 & 6.66 & 6.67 & 6.73 & 6.82 & 6.71 & 6.86 & 7.04 & 6.88 & 6.89 \\
\hline$p$-methylaniline & 7.04 & 6.98 & 6.97 & 7.02 & 7.09 & 7.01 & 7.11 & 7.30 & 7.19 & 7.19 \\
\hline N-methylaniline & 7.14 & 7.05 & 7.08 & 7.12 & 7.20 & 7.08 & 7.27 & 7.43 & 7.29 & 7.29 \\
\hline dimethylamine & 7.95 & 7.89 & 7.93 & 7.98 & 7.99 & 7.91 & 8.15 & 8.25 & 8.10 & 8.10 \\
\hline diethylamine & 7.59 & 7.50 & 7.61 & 7.62 & 7.65 & 7.53 & 7.87 & 7.93 & 7.76 & 7.76 \\
\hline pyrrolidine & 7.63 & 7.58 & 7.60 & 7.67 & 7.67 & 7.60 & 7.84 & 7.94 & 7.80 & 7.80 \\
\hline piperidine & 7.60 & 7.52 & 7.64 & 7.68 & 7.71 & 7.58 & 7.90 & 7.96 & 7.82 & 7.83 \\
\hline anisole & 7.91 & 7.81 & 7.84 & 7.88 & 7.98 & 7.85 & 7.99 & 8.19 & 8.04 & 8.04 \\
\hline indole & 7.48 & 7.39 & 7.40 & 7.45 & 7.55 & 7.43 & 7.55 & 7.78 & 7.61 & 7.62 \\
\hline$p$-methoxyphenol & 7.38 & 7.31 & 7.31 & 7.41 & 7.50 & 7.37 & 7.51 & 7.74 & 7.56 & 7.57 \\
\hline$p$-methylphenol & 7.79 & 7.74 & 7.71 & 7.80 & 7.87 & 7.78 & 7.85 & 8.08 & 7.95 & 7.96 \\
\hline phenol & 9.26 & 9.19 & 9.21 & 9.21 & 9.31 & 9.22 & 9.21 & 9.47 & 9.41 & 9.41 \\
\hline$p$-cyanophenol & 8.58 & 8.52 & 8.51 & 8.62 & 8.73 & 8.58 & 8.70 & 8.98 & 8.83 & 8.84 \\
\hline dimethylsulfide & 8.33 & 8.36 & 8.38 & 8.37 & 8.39 & 8.37 & 8.51 & 8.58 & 8.45 & 8.45 \\
\hline dimethyldisulfide & 7.70 & 7.72 & 7.78 & 7.77 & 7.83 & 7.77 & 7.94 & 8.03 & 7.84 & 7.86 \\
\hline thioanisole & 7.55 & 7.51 & 7.56 & 7.56 & 7.65 & 7.56 & 7.70 & 7.89 & 7.72 & 7.72 \\
\hline$p$-chloroaniline & 7.34 & 7.30 & 7.32 & 7.37 & 7.45 & 7.35 & 7.47 & 7.68 & 7.54 & 7.54 \\
\hline$p$-chlorophenol & 8.00 & 7.97 & 7.97 & 8.06 & 8.14 & 8.03 & 8.12 & 8.38 & 8.21 & 8.22 \\
\hline
\end{tabular}


Table S12. Difference in solvation energies $(\mathrm{eV})$ of reduced and oxidized forms calculated by ten density functionals combined with SMD.

\begin{tabular}{|c|c|c|c|c|c|c|c|c|c|c|c|c|c|}
\hline & $\mathrm{PBE}$ & TPSS & M06-L & B3LYP & PBE0 & TPSSh & M06 & M06-2X & B2PLYP & mPW2PLYP & MUE & MSE & $\mathrm{REF}$ \\
\hline aniline & -2.40 & -2.42 & -2.42 & -2.43 & -2.40 & -2.42 & -2.40 & -2.40 & -2.43 & -2.44 & 0.07 & 0.07 & -2.49 \\
\hline$p$-methoxyaniline & -2.13 & -2.14 & -2.15 & -2.16 & -2.14 & -2.15 & -2.14 & -2.15 & -2.16 & -2.16 & 0.06 & -0.06 & -2.09 \\
\hline$p$-methylaniline & -2.27 & -2.29 & -2.29 & -2.31 & -2.27 & -2.29 & -2.28 & -2.28 & -2.31 & -2.32 & 0.02 & 0.01 & -2.30 \\
\hline N-methylaniline & -2.33 & -2.33 & -2.34 & -2.37 & -2.34 & -2.34 & -2.35 & -2.36 & -2.37 & -2.38 & 0.06 & -0.06 & -2.29 \\
\hline dimethylamine & -2.77 & -2.76 & -2.78 & -2.79 & -2.78 & -2.77 & -2.78 & -2.77 & -2.79 & -2.79 & 0.03 & 0.03 & -2.81 \\
\hline diethylamine & -2.47 & -2.44 & -2.48 & -2.50 & -2.49 & -2.46 & -2.50 & -2.46 & -2.49 & -2.50 & 0.11 & -0.11 & -2.37 \\
\hline pyrrolidine & -2.58 & -2.58 & -2.60 & -2.61 & -2.60 & -2.59 & -2.60 & -2.59 & -2.61 & -2.62 & 0.07 & -0.07 & -2.53 \\
\hline piperidine & -2.41 & -2.41 & -2.43 & -2.45 & -2.44 & -2.43 & -2.44 & -2.43 & -2.45 & -2.47 & 0.05 & -0.05 & -2.39 \\
\hline anisole & -2.22 & -2.21 & -2.23 & -2.24 & -2.21 & -2.21 & -2.24 & -2.21 & -2.23 & -2.23 & 0.12 & 0.12 & -2.34 \\
\hline indole & -2.19 & -2.19 & -2.20 & -2.22 & -2.19 & -2.19 & -2.21 & -2.20 & -2.21 & -2.21 & 0.20 & 0.20 & -2.40 \\
\hline$p$-methoxyphenol & -2.18 & -2.17 & -2.19 & -2.20 & -2.18 & -2.17 & -2.21 & -2.20 & -2.20 & -2.21 & 0.05 & -0.05 & -2.14 \\
\hline$p$-methylphenol & -2.26 & -2.26 & -2.27 & -2.29 & -2.25 & -2.26 & -2.28 & -2.26 & -2.28 & -2.29 & 0.24 & 0.24 & -2.51 \\
\hline phenol & -2.32 & -2.31 & -2.33 & -2.33 & -2.31 & -2.31 & -2.33 & -2.30 & -2.32 & -2.32 & 0.44 & 0.44 & -2.76 \\
\hline$p$-cyanophenol & -2.47 & -2.47 & -2.49 & -2.53 & -2.51 & -2.49 & -2.54 & -2.54 & -2.55 & -2.56 & 0.57 & 0.57 & -3.08 \\
\hline dimethylsulfide & -2.81 & -2.80 & -2.80 & -2.83 & -2.81 & -2.80 & -2.83 & -2.83 & -2.84 & -2.84 & 0.23 & 0.23 & -3.05 \\
\hline dimethyldisulfide & -2.51 & -2.49 & -2.49 & -2.52 & -2.50 & -2.49 & -2.52 & -2.52 & -2.53 & -2.53 & 0.39 & -0.39 & -2.12 \\
\hline thioanisole & -2.21 & -2.19 & -2.20 & -2.23 & -2.20 & -2.19 & -2.22 & -2.21 & -2.23 & -2.23 & 0.01 & 0.00 & -2.21 \\
\hline$p$-chloroaniline & -2.35 & -2.37 & -2.38 & -2.40 & -2.37 & -2.38 & -2.37 & -2.39 & -2.40 & -2.41 & 0.39 & 0.39 & -2.77 \\
\hline$p$-chlorophenol & -2.33 & -2.33 & -2.35 & -2.37 & -2.34 & -2.34 & -2.36 & -2.35 & -2.36 & -2.37 & 1.11 & 1.11 & -3.46 \\
\hline MUE & 0.22 & 0.22 & 0.22 & 0.22 & 0.23 & 0.22 & 0.22 & 0.22 & 0.22 & 0.22 & & & \\
\hline MSE & 0.15 & 0.16 & 0.14 & 0.12 & 0.15 & 0.15 & 0.13 & 0.14 & 0.12 & 0.12 & & & \\
\hline
\end{tabular}


Table S13. Differences in solvation energies $(\mathrm{eV})$ of reduced and oxidized form calculated by ten density functionals combined with COSMO.

\begin{tabular}{|c|c|c|c|c|c|c|c|c|c|c|c|c|c|}
\hline & PBE & TPSS & M06-L & B3LYP & PBE0 & TPSSh & M06 & M06-2X & B2PLYP & mPW2PLYP & MUE & MSE & REF \\
\hline aniline & -2.21 & -2.23 & -2.24 & -2.23 & -2.22 & -2.23 & -2.22 & -2.22 & -2.24 & -2.24 & 0.26 & 0.26 & -2.49 \\
\hline$p$-methoxyaniline & -1.99 & -2.00 & -2.02 & -2.01 & -2.00 & -2.01 & -2.00 & -2.00 & -2.01 & -2.01 & 0.08 & 0.08 & -2.09 \\
\hline$p$-methylaniline & -2.10 & -2.12 & -2.13 & -2.13 & -2.11 & -2.12 & -2.11 & -2.11 & -2.13 & -2.14 & 0.18 & 0.18 & -2.30 \\
\hline $\mathrm{N}$-methylaniline & -2.14 & -2.15 & -2.16 & -2.16 & -2.15 & -2.15 & -2.15 & -2.15 & -2.17 & -2.17 & 0.14 & 0.14 & -2.29 \\
\hline dimethylamine & -2.67 & -2.67 & -2.69 & -2.69 & -2.69 & -2.68 & -2.69 & -2.68 & -2.69 & -2.70 & 0.13 & 0.13 & -2.81 \\
\hline diethylamine & -2.41 & -2.38 & -2.42 & -2.43 & -2.43 & -2.39 & -2.44 & -2.40 & -2.42 & -2.43 & 0.04 & -0.04 & -2.37 \\
\hline pyrrolidine & -2.49 & -2.49 & -2.50 & -2.51 & -2.51 & -2.50 & -2.51 & -2.50 & -2.51 & -2.52 & 0.03 & 0.03 & -2.53 \\
\hline piperidine & -2.34 & -2.35 & -2.36 & -2.38 & -2.38 & -2.36 & -2.37 & -2.37 & -2.38 & -2.39 & 0.02 & 0.02 & -2.39 \\
\hline anisole & -2.04 & -2.04 & -2.06 & -2.05 & -2.04 & -2.04 & -2.06 & -2.04 & -2.05 & -2.05 & 0.29 & 0.29 & -2.34 \\
\hline indole & -2.00 & -2.01 & -2.02 & -2.03 & -2.01 & -2.01 & -2.03 & -2.02 & -2.02 & -2.02 & 0.38 & 0.38 & -2.40 \\
\hline$p$-methoxyphenol & -1.97 & -1.96 & -1.99 & -1.98 & -1.97 & -1.97 & -1.99 & -1.97 & -1.98 & -1.98 & 0.16 & 0.16 & -2.14 \\
\hline$p$-methylphenol & -2.09 & -2.09 & -2.11 & -2.11 & -2.09 & -2.09 & -2.11 & -2.09 & -2.10 & -2.11 & 0.41 & 0.41 & -2.51 \\
\hline phenol & -1.11 & -1.08 & -1.09 & -1.13 & -1.10 & -1.09 & -1.20 & -1.13 & -1.08 & -1.08 & 1.65 & 1.65 & -2.76 \\
\hline$p$-cyanophenol & -2.23 & -2.24 & -2.26 & -2.28 & -2.27 & -2.25 & -2.29 & -2.30 & -2.30 & -2.30 & 0.81 & 0.81 & -3.08 \\
\hline dimethylsulfide & -2.52 & -2.51 & -2.52 & -2.52 & -2.52 & -2.51 & -2.53 & -2.53 & -2.53 & -2.53 & 0.53 & 0.53 & -3.05 \\
\hline dimethyldisulfide & -2.23 & -2.23 & -2.24 & -2.24 & -2.24 & -2.23 & -2.25 & -2.24 & -2.25 & -2.25 & 0.12 & -0.12 & -2.12 \\
\hline thioanisole & -1.98 & -1.98 & -1.99 & -1.99 & -1.98 & -1.98 & -2.00 & -1.99 & -2.00 & -2.00 & 0.22 & 0.22 & -2.21 \\
\hline$p$-chloroaniline & -2.13 & -2.16 & -2.17 & -2.17 & -2.16 & -2.17 & -2.15 & -2.17 & -2.17 & -2.18 & 0.61 & 0.61 & -2.77 \\
\hline$p$-chlorophenol & -2.11 & -2.12 & -2.14 & -2.14 & -2.13 & -2.12 & -2.14 & -2.13 & -2.13 & -2.14 & 1.33 & 1.33 & -3.46 \\
\hline MUE & 0.40 & 0.40 & 0.39 & 0.38 & 0.39 & 0.39 & 0.38 & 0.39 & 0.38 & 0.38 & & & \\
\hline MSE & 0.39 & 0.38 & 0.37 & 0.36 & 0.37 & 0.38 & 0.36 & 0.37 & 0.37 & 0.36 & & & \\
\hline
\end{tabular}


Table S14. Solvation energies (eV) of reduced form and statistical errors calculated for ten density functionals combined with SMD.

\begin{tabular}{|c|c|c|c|c|c|c|c|c|c|c|c|c|c|}
\hline & $\mathrm{PBE}$ & TPSS & M06-L & B3LYP & PBE0 & TPSSh & M06 & M06- & B2PLY & mPW2PLY & MUE & MSE & REF \\
\hline aniline & -0.17 & -0.15 & -0.13 & -0.15 & -0.19 & -0.16 & -0.16 & -0.18 & -0.15 & -0.15 & 0.08 & 0.08 & -0.24 \\
\hline$p$-methoxyaniline & -0.18 & -0.17 & -0.13 & -0.18 & -0.20 & -0.18 & -0.17 & -0.20 & -0.17 & -0.18 & 0.15 & 0.15 & -0.33 \\
\hline$p$-methylaniline & -0.14 & -0.12 & -0.09 & -0.12 & -0.16 & -0.12 & -0.13 & -0.15 & -0.12 & -0.12 & 0.14 & 0.14 & -0.27 \\
\hline $\mathrm{N}$-methylaniline & -0.06 & -0.05 & -0.02 & -0.04 & -0.07 & -0.05 & -0.03 & -0.04 & -0.03 & -0.03 & 0.16 & 0.16 & -0.20 \\
\hline dimethylamine & -0.20 & -0.20 & -0.19 & -0.20 & -0.21 & -0.21 & -0.20 & -0.22 & -0.22 & -0.22 & 0.04 & -0.04 & -0.17 \\
\hline diethylamine & -0.17 & -0.18 & -0.18 & -0.17 & -0.19 & -0.18 & -0.18 & -0.21 & -0.19 & -0.19 & 0.01 & 0.00 & -0.18 \\
\hline pyrrolidine & -0.23 & -0.23 & -0.22 & -0.23 & -0.24 & -0.23 & -0.23 & -0.25 & -0.24 & -0.24 & 0.01 & 0.01 & -0.24 \\
\hline piperidine & -0.20 & -0.21 & -0.20 & -0.21 & -0.22 & -0.21 & -0.21 & -0.23 & -0.22 & -0.22 & 0.02 & 0.02 & -0.23 \\
\hline anisole & -0.06 & -0.06 & -0.02 & -0.06 & -0.07 & -0.06 & -0.04 & -0.09 & -0.07 & -0.07 & 0.05 & 0.05 & -0.11 \\
\hline indole & -0.21 & -0.19 & -0.16 & -0.19 & -0.23 & -0.20 & -0.18 & -0.23 & -0.20 & -0.21 & 0.05 & 0.05 & -0.25 \\
\hline$p$-methoxyphenol & -0.24 & -0.24 & -0.20 & -0.25 & -0.26 & -0.25 & -0.23 & -0.28 & -0.26 & -0.26 & 0.02 & 0.00 & -0.25 \\
\hline$p$-methylphenol & -0.18 & -0.17 & -0.14 & -0.18 & -0.20 & -0.18 & -0.17 & -0.21 & -0.19 & -0.19 & 0.08 & 0.08 & -0.26 \\
\hline phenol & -0.20 & -0.19 & -0.16 & -0.20 & -0.22 & -0.20 & -0.19 & -0.23 & -0.21 & -0.21 & 0.09 & 0.09 & -0.29 \\
\hline$p$-cyanophenol & -0.36 & -0.36 & -0.35 & -0.38 & -0.38 & -0.37 & -0.36 & -0.38 & -0.36 & -0.37 & 0.08 & 0.08 & -0.45 \\
\hline dimethylsulfide & -0.05 & -0.05 & -0.04 & -0.05 & -0.06 & -0.06 & -0.03 & -0.05 & -0.05 & -0.05 & 0.02 & 0.02 & -0.07 \\
\hline dimethyldisulfide & -0.08 & -0.08 & -0.07 & -0.08 & -0.09 & -0.09 & -0.07 & -0.09 & -0.08 & -0.09 & 0.01 & -0.01 & -0.07 \\
\hline thioanisole & -0.08 & -0.07 & -0.05 & -0.07 & -0.10 & -0.08 & -0.06 & -0.10 & -0.08 & -0.08 & 0.04 & 0.04 & -0.12 \\
\hline$p$-chloroaniline & -0.19 & -0.18 & -0.16 & -0.18 & -0.21 & -0.18 & -0.18 & -0.20 & -0.17 & -0.17 & 0.08 & 0.08 & -0.26 \\
\hline$p$-chlorophenol & -0.21 & -0.21 & -0.19 & -0.22 & -0.23 & -0.22 & -0.21 & -0.24 & -0.22 & -0.23 & 0.17 & 0.17 & -0.39 \\
\hline MUE & 0.07 & 0.07 & 0.09 & 0.07 & 0.05 & 0.07 & 0.07 & 0.06 & 0.07 & 0.07 & & & \\
\hline MSE & 0.06 & 0.07 & 0.09 & 0.06 & 0.04 & 0.06 & 0.07 & 0.04 & 0.06 & 0.06 & & & \\
\hline
\end{tabular}


Table S15. Solvation energies (eV) of reduced forms and statistical errors calculated for ten density functionals combined with COSMO.

\begin{tabular}{|c|c|c|c|c|c|c|c|c|c|c|c|c|c|}
\hline & PBE & TPSS & M06-L & B3LYP & PBE0 & TPSSh & M06 & M06-2X & B2PLYP & mPW2PLYP & MUE & MSE & REF \\
\hline aniline & -0.25 & -0.23 & -0.21 & -0.24 & -0.26 & -0.24 & -0.24 & -0.26 & -0.23 & -0.24 & 0.01 & 0.00 & -0.24 \\
\hline$p$-methoxyaniline & -0.29 & -0.27 & -0.24 & -0.28 & -0.30 & -0.28 & -0.28 & -0.30 & -0.28 & -0.28 & 0.05 & 0.05 & -0.33 \\
\hline$p$-methylaniline & -0.24 & -0.22 & -0.20 & -0.22 & -0.25 & -0.22 & -0.23 & -0.24 & -0.22 & -0.22 & 0.04 & 0.04 & -0.27 \\
\hline $\mathrm{N}$-methylaniline & -0.16 & -0.14 & -0.12 & -0.14 & -0.16 & -0.14 & -0.13 & -0.13 & -0.12 & -0.12 & 0.06 & 0.06 & -0.20 \\
\hline dimethylamine & -0.13 & -0.13 & -0.13 & -0.13 & -0.14 & -0.14 & -0.13 & -0.15 & -0.14 & -0.14 & 0.03 & 0.03 & -0.17 \\
\hline diethylamine & -0.13 & -0.13 & -0.13 & -0.12 & -0.14 & -0.13 & -0.13 & -0.15 & -0.14 & -0.14 & 0.05 & 0.05 & -0.18 \\
\hline pyrrolidine & -0.13 & -0.13 & -0.13 & -0.13 & -0.14 & -0.13 & -0.13 & -0.15 & -0.14 & -0.14 & 0.10 & 0.10 & -0.24 \\
\hline piperidine & -0.13 & -0.13 & -0.12 & -0.13 & -0.13 & -0.13 & -0.13 & -0.14 & -0.13 & -0.13 & 0.10 & 0.10 & -0.23 \\
\hline anisole & -0.19 & -0.19 & -0.15 & -0.19 & -0.20 & -0.19 & -0.17 & -0.21 & -0.20 & -0.20 & 0.08 & -0.08 & -0.11 \\
\hline indole & -0.30 & -0.29 & -0.27 & -0.29 & -0.32 & -0.30 & -0.28 & -0.32 & -0.31 & -0.31 & 0.05 & -0.05 & -0.25 \\
\hline$p$-methoxyphenol & -0.33 & -0.34 & -0.30 & -0.34 & -0.34 & -0.34 & -0.32 & -0.36 & -0.35 & -0.35 & 0.09 & -0.09 & -0.25 \\
\hline$p$-methylphenol & -0.27 & -0.26 & -0.24 & -0.27 & -0.28 & -0.27 & -0.26 & -0.29 & -0.28 & -0.28 & 0.01 & -0.01 & -0.26 \\
\hline phenol & -0.27 & -0.27 & -0.24 & -0.27 & -0.28 & -0.27 & -0.26 & -0.29 & -0.28 & -0.28 & 0.02 & 0.02 & -0.29 \\
\hline$p$-cyanophenol & -0.47 & -0.47 & -0.45 & -0.48 & -0.48 & -0.47 & -0.46 & -0.48 & -0.47 & -0.47 & 0.02 & -0.02 & -0.45 \\
\hline dimethylsulfide & -0.14 & -0.14 & -0.13 & -0.14 & -0.15 & -0.14 & -0.13 & -0.14 & -0.14 & -0.14 & 0.07 & -0.07 & -0.07 \\
\hline dimethyldisulfide & -0.16 & -0.16 & -0.15 & -0.16 & -0.17 & -0.16 & -0.15 & -0.17 & -0.16 & -0.16 & 0.09 & -0.09 & -0.07 \\
\hline thioanisole & -0.20 & -0.19 & -0.17 & -0.19 & -0.21 & -0.20 & -0.18 & -0.22 & -0.20 & -0.20 & 0.08 & -0.08 & -0.12 \\
\hline$p$-chloroaniline & -0.28 & -0.26 & -0.25 & -0.27 & -0.29 & -0.27 & -0.27 & -0.28 & -0.26 & -0.26 & 0.01 & -0.01 & -0.26 \\
\hline$p$-chlorophenol & -0.28 & -0.28 & -0.26 & -0.29 & -0.30 & -0.29 & -0.28 & -0.30 & -0.29 & -0.29 & 0.10 & 0.10 & -0.39 \\
\hline MUE & 0.06 & 0.06 & 0.06 & 0.06 & 0.06 & 0.06 & 0.05 & 0.06 & 0.06 & 0.06 & & & \\
\hline MSE & 0.00 & 0.01 & 0.03 & 0.01 & -0.01 & 0.00 & 0.01 & -0.01 & 0.00 & 0.00 & & & \\
\hline
\end{tabular}


Table S16. Solvation energies (eV) of the oxidized forms and statistical errors calculated for ten density functionals combined with SMD.

\begin{tabular}{|c|c|c|c|c|c|c|c|c|c|c|c|c|c|}
\hline & $\mathrm{PBE}$ & TPSS & M06-L & B3LYP & PBE0 & TPSSh & M06 & M06-2X & B2PLYP & mPW2PLYP & MUE & MSE & REF \\
\hline aniline & -2.57 & -2.57 & -2.55 & -2.58 & -2.59 & -2.57 & -2.56 & -2.58 & -2.58 & -2.59 & 0.16 & 0.16 & -2.73 \\
\hline$p$-methoxyaniline & -2.32 & -2.31 & -2.29 & -2.34 & -2.34 & -2.32 & -2.32 & -2.35 & -2.33 & -2.34 & 0.09 & 0.09 & -2.42 \\
\hline$p$-methylaniline & -2.41 & -2.41 & -2.39 & -2.43 & -2.43 & -2.42 & -2.41 & -2.43 & -2.43 & -2.44 & 0.15 & 0.15 & -2.57 \\
\hline N-methylaniline & -2.39 & -2.38 & -2.37 & -2.40 & -2.41 & -2.39 & -2.38 & -2.40 & -2.40 & -2.41 & 0.10 & 0.10 & -2.49 \\
\hline dimethylamine & -2.97 & -2.97 & -2.97 & -2.99 & -2.99 & -2.98 & -2.98 & -2.99 & -3.00 & -3.01 & 0.01 & -0.01 & -2.98 \\
\hline diethylamine & -2.65 & -2.62 & -2.66 & -2.67 & -2.68 & -2.64 & -2.68 & -2.67 & -2.68 & -2.69 & 0.11 & -0.11 & -2.55 \\
\hline pyrrolidine & -2.81 & -2.81 & -2.82 & -2.84 & -2.84 & -2.82 & -2.83 & -2.84 & -2.85 & -2.86 & 0.06 & -0.06 & -2.77 \\
\hline piperidine & -2.61 & -2.62 & -2.63 & -2.66 & -2.66 & -2.64 & -2.65 & -2.66 & -2.67 & -2.68 & 0.03 & -0.03 & -2.62 \\
\hline anisole & -2.28 & -2.27 & -2.25 & -2.30 & -2.28 & -2.27 & -2.28 & -2.30 & -2.30 & -2.31 & 0.17 & 0.17 & -2.45 \\
\hline indole & -2.39 & -2.38 & -2.36 & -2.41 & -2.42 & -2.39 & -2.39 & -2.43 & -2.41 & -2.42 & 0.25 & 0.25 & -2.65 \\
\hline$p$-methoxyphenol & -2.41 & -2.41 & -2.39 & -2.45 & -2.44 & -2.42 & -2.44 & -2.48 & -2.46 & -2.47 & 0.05 & -0.05 & -2.39 \\
\hline$p$-methylphenol & -2.44 & -2.43 & -2.42 & -2.46 & -2.45 & -2.44 & -2.45 & -2.47 & -2.47 & -2.47 & 0.32 & 0.32 & -2.77 \\
\hline phenol & -2.52 & -2.51 & -2.50 & -2.53 & -2.52 & -2.51 & -2.52 & -2.53 & -2.53 & -2.53 & 0.53 & 0.53 & -3.05 \\
\hline p-cyanophenol & -2.83 & -2.84 & -2.84 & -2.91 & -2.89 & -2.86 & -2.90 & -2.92 & -2.92 & -2.93 & 0.65 & 0.65 & -3.53 \\
\hline dimethylsulfide & -2.85 & -2.85 & -2.84 & -2.88 & -2.87 & -2.85 & -2.87 & -2.88 & -2.89 & -2.89 & 0.25 & 0.25 & -3.12 \\
\hline dimethyldisulfide & -2.58 & -2.57 & -2.56 & -2.60 & -2.59 & -2.58 & -2.59 & -2.61 & -2.61 & -2.61 & 0.40 & -0.40 & -2.19 \\
\hline thioanisole & -2.28 & -2.27 & -2.25 & -2.30 & -2.30 & -2.27 & -2.28 & -2.31 & -2.30 & -2.31 & 0.04 & 0.04 & -2.33 \\
\hline$p$-chloroaniline & -2.54 & -2.55 & -2.53 & -2.58 & -2.58 & -2.56 & -2.55 & -2.58 & -2.57 & -2.58 & 0.46 & 0.46 & -3.02 \\
\hline$p$-chlorophenol & -2.54 & -2.55 & -2.54 & -2.59 & -2.57 & -2.56 & -2.57 & -2.59 & -2.58 & -2.59 & 1.27 & 1.27 & -3.84 \\
\hline MUE & 0.27 & 0.27 & 0.28 & 0.26 & 0.26 & 0.27 & 0.27 & 0.26 & 0.26 & 0.26 & & & \\
\hline MSE & 0.21 & 0.22 & 0.23 & 0.19 & 0.19 & 0.21 & 0.20 & 0.18 & 0.18 & 0.18 & & & \\
\hline
\end{tabular}


Table S17. Solvation energies (eV) of the oxidized forms and statistical errors calculated for ten density functionals combined with COSMO.

\begin{tabular}{|c|c|c|c|c|c|c|c|c|c|c|c|c|c|}
\hline & $\mathrm{PBE}$ & TPSS & M06-L & B3LYP & PBE0 & TPSSh & M06 & M06-2X & B2PLYP & mPW2PLYP & MUE & MSE & REF \\
\hline aniline & -2.47 & -2.46 & -2.45 & -2.47 & -2.49 & -2.47 & -2.46 & -2.48 & -2.47 & -2.48 & 0.26 & 0.26 & -2.73 \\
\hline$p$-methoxyaniline & -2.28 & -2.27 & -2.26 & -2.29 & -2.30 & -2.28 & -2.28 & -2.30 & -2.29 & -2.29 & 0.14 & 0.14 & -2.42 \\
\hline$p$-methylaniline & -2.34 & -2.34 & -2.33 & -2.35 & -2.36 & -2.35 & -2.34 & -2.36 & -2.35 & -2.36 & 0.22 & 0.22 & -2.57 \\
\hline $\mathrm{N}$-methylaniline & -2.30 & -2.29 & -2.28 & -2.30 & -2.31 & -2.30 & -2.28 & -2.28 & -2.29 & -2.30 & 0.20 & 0.20 & -2.49 \\
\hline dimethylamine & -2.80 & -2.80 & -2.81 & -2.82 & -2.83 & -2.81 & -2.82 & -2.83 & -2.83 & -2.84 & 0.16 & 0.16 & -2.98 \\
\hline diethylamine & -2.53 & -2.51 & -2.56 & -2.56 & -2.57 & -2.53 & -2.57 & -2.56 & -2.56 & -2.57 & 0.02 & 0.00 & -2.55 \\
\hline pyrrolidine & -2.62 & -2.62 & -2.63 & -2.64 & -2.65 & -2.63 & -2.64 & -2.64 & -2.65 & -2.66 & 0.13 & 0.13 & -2.77 \\
\hline piperidine & -2.47 & -2.47 & -2.49 & -2.50 & -2.51 & -2.49 & -2.50 & -2.51 & -2.52 & -2.53 & 0.12 & 0.12 & -2.62 \\
\hline anisole & -2.23 & -2.22 & -2.21 & -2.24 & -2.24 & -2.23 & -2.23 & -2.24 & -2.24 & -2.25 & 0.22 & 0.22 & -2.45 \\
\hline indole & -2.31 & -2.30 & -2.29 & -2.32 & -2.34 & -2.31 & -2.31 & -2.34 & -2.32 & -2.33 & 0.33 & 0.33 & -2.65 \\
\hline$p$-methoxyphenol & -2.30 & -2.30 & -2.29 & -2.32 & -2.32 & -2.31 & -2.31 & -2.33 & -2.32 & -2.33 & 0.08 & 0.08 & -2.39 \\
\hline$p$-methylphenol & -2.36 & -2.35 & -2.34 & -2.37 & -2.37 & -2.36 & -2.37 & -2.38 & -2.38 & -2.38 & 0.40 & 0.40 & -2.77 \\
\hline phenol & -1.38 & -1.35 & -1.33 & -1.40 & -1.38 & -1.36 & -1.46 & -1.42 & -1.35 & -1.36 & 1.67 & 1.67 & -3.05 \\
\hline p-cyanophenol & -2.70 & -2.71 & -2.71 & -2.75 & -2.75 & -2.73 & -2.75 & -2.77 & -2.77 & -2.78 & 0.79 & 0.79 & -3.53 \\
\hline dimethylsulfide & -2.65 & -2.65 & -2.65 & -2.66 & -2.66 & -2.65 & -2.66 & -2.67 & -2.67 & -2.67 & 0.46 & 0.46 & -3.12 \\
\hline dimethyldisulfide & -2.39 & -2.38 & -2.38 & -2.40 & -2.40 & -2.39 & -2.39 & -2.41 & -2.40 & -2.41 & 0.21 & -0.21 & -2.19 \\
\hline thioanisole & -2.18 & -2.17 & -2.16 & -2.19 & -2.20 & -2.18 & -2.18 & -2.20 & -2.20 & -2.20 & 0.14 & 0.14 & -2.33 \\
\hline$p$-chloroaniline & -2.41 & -2.42 & -2.41 & -2.44 & -2.45 & -2.43 & -2.42 & -2.45 & -2.43 & -2.44 & 0.59 & 0.59 & -3.02 \\
\hline$p$-chlorophenol & -2.40 & -2.40 & -2.40 & -2.43 & -2.42 & -2.41 & -2.42 & -2.43 & -2.42 & -2.43 & 1.42 & 1.42 & -3.84 \\
\hline MUE & 0.41 & 0.41 & 0.42 & 0.39 & 0.39 & 0.40 & 0.40 & 0.39 & 0.39 & 0.39 & & & \\
\hline MSE & 0.39 & 0.39 & 0.39 & 0.37 & 0.36 & 0.38 & 0.37 & 0.36 & 0.37 & 0.36 & & & \\
\hline
\end{tabular}


Table S18. Redox potentials obtained for selected wave function methods.

\begin{tabular}{|c|c|c|c|c|c|c|c|c|c|c|c|}
\hline & $\operatorname{CCSD}(\mathrm{T})$ & LPNO-pCCSD & OO-MP2 & CCSD & LPNO-CCSD & LPNO-CEPA & $\mathrm{F} 12-\mathrm{CCSD}(\mathrm{T})$ & SCS-MP2 & F12-MP2 & MP2 & $\mathrm{HF}$ \\
\hline & cc-pV[D/T]Z & cc-pV[T/Q]Z & cc-pV[T/Q]Z & cc-pV[D/T]Z & cc-pV[T/Q]Z & cc-pV[T/Q]Z & cc-pVDZ-F12 & cc-pV[T/Q]Z & cc-pVTZ-F12 & cc-pV[T/Q]Z & cc-pVTZ \\
\hline aniline & 1.08 & 1.03 & 0.96 & 0.95 & 0.99 & 0.95 & 0.95 & 1.90 & 1.95 & 2.46 & -0.55 \\
\hline$p$-methoxyaniline & 0.73 & 0.73 & 0.75 & 0.64 & 0.66 & 0.61 & 0.58 & 1.35 & 1.35 & 1.45 & -0.61 \\
\hline$p$-methylaniline & 0.86 & 0.81 & 0.93 & 0.73 & 0.75 & 0.71 & 0.71 & 1.53 & 1.67 & 1.66 & -0.70 \\
\hline N-methylaniline & 0.86 & 0.81 & 0.93 & 0.73 & 0.77 & 0.73 & 0.71 & 1.71 & 1.85 & 1.84 & -0.79 \\
\hline dimethylamine & 1.28 & 1.25 & 1.37 & 1.18 & 1.19 & 1.18 & 1.18 & 1.45 & 1.49 & 1.48 & -0.20 \\
\hline diethylamine & 1.27 & 1.25 & 1.35 & 1.18 & 1.20 & 1.18 & 1.15 & 1.45 & 1.50 & 1.49 & -0.23 \\
\hline pyrrolidine & 1.17 & 1.14 & 1.27 & 1.07 & 1.08 & 1.07 & 1.05 & 1.36 & 1.40 & 1.39 & -0.32 \\
\hline piperidine & 1.36 & 1.37 & 1.38 & 1.29 & 1.34 & 1.30 & 1.23 & 1.59 & 1.62 & 1.61 & 0.02 \\
\hline anisole & 1.77 & 1.75 & 1.85 & 1.65 & 1.69 & 1.65 & 1.63 & 2.35 & 2.51 & 2.50 & 0.23 \\
\hline indole & 1.37 & 1.33 & 1.42 & 1.26 & 1.30 & 1.59 & 1.22 & 2.31 & 2.49 & 2.48 & -0.26 \\
\hline$p$-methoxyphenol & 1.28 & 1.29 & 1.32 & 1.20 & 1.23 & 1.18 & 1.13 & 1.54 & 1.65 & 1.65 & -0.02 \\
\hline$p$-methylphenol & 1.66 & 1.64 & 1.75 & 1.54 & 1.56 & 1.54 & 1.51 & 2.09 & 2.23 & 2.22 & 0.20 \\
\hline phenol & 1.96 & 1.93 & 2.06 & 1.83 & 1.86 & 1.84 & 1.82 & 2.49 & 2.65 & 2.64 & 0.40 \\
\hline$p$-cyanophenol & 2.32 & 2.28 & 2.35 & 2.21 & 2.25 & 2.18 & 2.14 & 3.48 & 3.61 & 3.61 & 0.74 \\
\hline dimethylsulfide & 1.59 & 1.58 & 1.60 & 1.51 & 1.52 & 1.52 & 1.43 & 1.64 & 1.66 & 1.65 & 0.35 \\
\hline dimethyldisulfide & 1.33 & 1.34 & 1.26 & 1.31 & 1.35 & 1.33 & 1.15 & 1.38 & 1.35 & 1.34 & 0.45 \\
\hline thioanisole & 1.54 & 1.49 & 1.51 & 1.45 & 1.46 & 1.42 & 1.34 & 2.31 & 2.40 & 2.40 & 0.12 \\
\hline$p$-chloroaniline & 1.15 & 1.34 & 1.17 & 1.03 & 1.31 & 1.27 & 0.97 & 1.92 & 2.04 & 2.04 & -0.33 \\
\hline$p$-chlorophenol & 1.87 & 1.37 & 1.90 & 1.78 & 1.34 & 1.30 & 1.69 & 2.40 & 2.52 & 2.52 & 0.54 \\
\hline MUE & 0.28 & 0.28 & 0.30 & 0.29 & 0.28 & 0.30 & 0.30 & 0.69 & 0.78 & 0.80 & 1.29 \\
\hline MSE & 0.16 & 0.12 & 0.19 & 0.06 & 0.07 & 0.06 & 0.01 & 0.67 & 0.76 & 0.79 & -1.29 \\
\hline
\end{tabular}


Table S19. Redox potentials calculated for ten density functionals combined with SMD.

\begin{tabular}{|c|c|c|c|c|c|c|c|c|c|c|c|c|c|}
\hline & PBE & TPSS & M06-L & B3LYP & PBE0 & TPSSh & M06 & M06-2X & B2PLYP & mPW2PLYP & MUE & MSE & REF \\
\hline aniline & 0.76 & 0.66 & 0.68 & 0.70 & 0.80 & 0.69 & 0.83 & 1.01 & 0.86 & 0.85 & 0.24 & -0.24 & 1.02 \\
\hline$p$-methoxyaniline & 0.31 & 0.24 & 0.24 & 0.29 & 0.40 & 0.28 & 0.43 & 0.61 & 0.44 & 0.45 & 0.42 & -0.42 & 0.79 \\
\hline$p$-methylaniline & 0.48 & 0.40 & 0.40 & 0.43 & 0.54 & 0.43 & 0.55 & 0.74 & 0.60 & 0.59 & 0.40 & -0.40 & 0.92 \\
\hline N-methylaniline & 0.53 & 0.44 & 0.46 & 0.47 & 0.58 & 0.47 & 0.64 & 0.79 & 0.64 & 0.63 & 0.38 & -0.38 & 0.95 \\
\hline dimethylamine & 0.91 & 0.85 & 0.87 & 0.91 & 0.93 & 0.86 & 1.09 & 1.20 & 1.03 & 1.03 & 0.30 & -0.30 & 1.27 \\
\hline diethylamine & 0.84 & 0.78 & 0.85 & 0.84 & 0.87 & 0.79 & 1.09 & 1.19 & 0.99 & 0.98 & 0.44 & -0.44 & 1.36 \\
\hline pyrrolidine & 0.77 & 0.72 & 0.72 & 0.78 & 0.80 & 0.73 & 0.95 & 1.07 & 0.91 & 0.90 & 0.42 & -0.42 & 1.26 \\
\hline piperidine & 0.91 & 0.83 & 0.93 & 0.95 & 0.99 & 0.87 & 1.17 & 1.25 & 1.08 & 1.08 & 0.33 & -0.33 & 1.34 \\
\hline anisole & 1.41 & 1.32 & 1.33 & 1.36 & 1.49 & 1.37 & 1.47 & 1.70 & 1.53 & 1.53 & 0.19 & -0.17 & 1.62 \\
\hline indole & 1.02 & 0.92 & 0.92 & 0.95 & 1.09 & 0.96 & 1.06 & 1.30 & 1.13 & 1.12 & 0.10 & -0.03 & 1.08 \\
\hline$p$-methoxyphenol & 0.92 & 0.86 & 0.84 & 0.93 & 1.04 & 0.92 & 1.02 & 1.26 & 1.07 & 1.08 & 0.24 & -0.24 & 1.23 \\
\hline$p$-methylphenol & 1.25 & 1.20 & 1.15 & 1.23 & 1.34 & 1.24 & 1.29 & 1.54 & 1.39 & 1.39 & 0.11 & -0.08 & 1.38 \\
\hline phenol & 2.66 & 2.60 & 2.59 & 2.60 & 2.73 & 2.63 & 2.60 & 2.89 & 2.81 & 2.80 & 1.19 & 1.19 & 1.50 \\
\hline p-cyanophenol & 1.83 & 1.76 & 1.74 & 1.81 & 1.94 & 1.81 & 1.88 & 2.16 & 2.00 & 2.00 & 0.18 & 0.18 & 1.71 \\
\hline dimethylsulfide & 1.25 & 1.28 & 1.29 & 1.26 & 1.30 & 1.30 & 1.39 & 1.47 & 1.33 & 1.33 & 0.09 & -0.07 & 1.39 \\
\hline dimethyldisulfide & 0.91 & 0.95 & 1.01 & 0.97 & 1.04 & 1.00 & 1.14 & 1.23 & 1.03 & 1.05 & 0.65 & -0.65 & 1.68 \\
\hline thioanisole & 1.06 & 1.04 & 1.07 & 1.06 & 1.17 & 1.09 & 1.20 & 1.40 & 1.21 & 1.22 & 0.30 & -0.30 & 1.45 \\
\hline$p$-chloroaniline & 0.72 & 0.65 & 0.66 & 0.70 & 0.80 & 0.69 & 0.82 & 1.01 & 0.86 & 0.86 & 0.11 & 0.10 & 0.68 \\
\hline$p$-chlorophenol & 1.39 & 1.36 & 1.34 & 1.41 & 1.52 & 1.41 & 1.48 & 1.75 & 1.57 & 1.58 & 0.83 & 0.83 & 0.65 \\
\hline MUE & 0.39 & 0.43 & 0.41 & 0.40 & 0.36 & 0.41 & 0.30 & 0.28 & 0.33 & 0.33 & & & \\
\hline MSE & -0.18 & -0.23 & -0.22 & -0.19 & -0.10 & -0.20 & -0.06 & 0.12 & -0.04 & -0.04 & & & \\
\hline
\end{tabular}


Table S20. Redox potentials calculated for ten density functionals combined with COSMO.

\begin{tabular}{|c|c|c|c|c|c|c|c|c|c|c|c|c|c|}
\hline & $\mathrm{PBE}$ & TPSS & M06-L & B3LYP & PBE0 & TPSSh & M06 & M06-2X & B2PLYP & mPW2PLYP & MUE & MSE & REF \\
\hline aniline & 0.95 & 0.85 & 0.86 & 0.89 & 0.98 & 0.87 & 1.01 & 1.19 & 1.05 & 1.04 & 0.10 & -0.05 & 1.02 \\
\hline$p$-methoxyaniline & 0.46 & 0.38 & 0.38 & 0.45 & 0.54 & 0.42 & 0.58 & 0.75 & 0.59 & 0.60 & 0.28 & -0.28 & 0.79 \\
\hline$p$-methylaniline & 0.66 & 0.58 & 0.56 & 0.61 & 0.70 & 0.60 & 0.73 & 0.91 & 0.77 & 0.77 & 0.23 & -0.23 & 0.92 \\
\hline $\mathrm{N}$-methylaniline & 0.71 & 0.61 & 0.63 & 0.65 & 0.75 & 0.64 & 0.81 & 0.96 & 0.82 & 0.81 & 0.21 & -0.21 & 0.95 \\
\hline dimethylamine & 1.00 & 0.94 & 0.96 & 1.01 & 1.02 & 0.95 & 1.18 & 1.29 & 1.13 & 1.12 & 0.21 & -0.21 & 1.27 \\
\hline diethylamine & 0.90 & 0.84 & 0.90 & 0.91 & 0.93 & 0.85 & 1.15 & 1.25 & 1.05 & 1.05 & 0.38 & -0.38 & 1.36 \\
\hline pyrrolidine & 0.86 & 0.81 & 0.81 & 0.88 & 0.89 & 0.82 & 1.05 & 1.16 & 1.01 & 1.00 & 0.33 & -0.33 & 1.26 \\
\hline piperidine & 0.98 & 0.90 & 1.00 & 1.02 & 1.05 & 0.94 & 1.24 & 1.32 & 1.16 & 1.16 & 0.26 & -0.26 & 1.34 \\
\hline anisole & 1.59 & 1.50 & 1.50 & 1.55 & 1.66 & 1.54 & 1.65 & 1.87 & 1.71 & 1.71 & 0.09 & 0.01 & 1.62 \\
\hline indole & 1.20 & 1.10 & 1.10 & 1.14 & 1.26 & 1.14 & 1.24 & 1.48 & 1.32 & 1.31 & 0.15 & 0.15 & 1.08 \\
\hline$p$-methoxyphenol & 1.06 & 1.00 & 0.97 & 1.08 & 1.17 & 1.06 & 1.17 & 1.40 & 1.22 & 1.23 & 0.13 & -0.09 & 1.23 \\
\hline$p$-methylphenol & 1.42 & 1.37 & 1.32 & 1.41 & 1.50 & 1.41 & 1.46 & 1.71 & 1.57 & 1.57 & 0.11 & 0.09 & 1.38 \\
\hline phenol & 2.82 & 2.76 & 2.74 & 2.77 & 2.88 & 2.79 & 2.76 & 3.04 & 2.97 & 2.97 & 1.35 & 1.35 & 1.50 \\
\hline p-cyanophenol & 2.07 & 2.00 & 1.97 & 2.07 & 2.17 & 2.05 & 2.13 & 2.40 & 2.25 & 2.25 & 0.43 & 0.43 & 1.71 \\
\hline dimethylsulfide & 1.54 & 1.57 & 1.58 & 1.57 & 1.59 & 1.58 & 1.69 & 1.78 & 1.64 & 1.64 & 0.23 & 0.23 & 1.39 \\
\hline dimethyldisulfide & 1.19 & 1.21 & 1.27 & 1.25 & 1.31 & 1.26 & 1.41 & 1.51 & 1.31 & 1.33 & 0.37 & -0.37 & 1.68 \\
\hline thioanisole & 1.29 & 1.25 & 1.28 & 1.29 & 1.39 & 1.30 & 1.42 & 1.62 & 1.44 & 1.45 & 0.11 & -0.08 & 1.45 \\
\hline$p$-chloroaniline & 0.93 & 0.87 & 0.87 & 0.92 & 1.01 & 0.90 & 1.04 & 1.23 & 1.09 & 1.08 & 0.31 & 0.31 & 0.68 \\
\hline$p$-chlorophenol & 1.61 & 1.57 & 1.55 & 1.64 & 1.73 & 1.62 & 1.70 & 1.97 & 1.80 & 1.80 & 1.05 & 1.05 & 0.65 \\
\hline MUE & 0.34 & 0.36 & 0.35 & 0.34 & 0.33 & 0.35 & 0.27 & 0.34 & 0.32 & 0.32 & & & \\
\hline MSE & 0.00 & -0.06 & -0.05 & -0.01 & 0.07 & -0.03 & 0.11 & 0.29 & 0.14 & 0.14 & & & \\
\hline
\end{tabular}


Table S21. Solvation energies (eV) for phenol-water clusters.

\begin{tabular}{|c|c|c|c|c|c|c|c|c|c|}
\hline & & $\Delta \Delta G_{\mathrm{solv}}$ & & & $\Delta G_{\text {solv }}(\operatorname{Red})$ & & & $\Delta G_{\mathrm{solv}}(\mathrm{Ox})$ & \\
\hline & $\begin{array}{c}\mathrm{C}_{6} \mathrm{H}_{5} \mathrm{OH}- \\
\mathrm{H}_{2} \mathrm{O} \\
\end{array}$ & $\begin{array}{c}\mathrm{C}_{6} \mathrm{H}_{5} \mathrm{OH}- \\
\left(\mathrm{H}_{2} \mathrm{O}\right)_{2} \\
\end{array}$ & $\begin{array}{c}\mathrm{C}_{6} \mathrm{H}_{5} \mathrm{OH}- \\
\left(\mathrm{H}_{2} \mathrm{O}\right)_{3} \\
\end{array}$ & $\begin{array}{c}\mathrm{C}_{6} \mathrm{H}_{5} \mathrm{OH}- \\
\mathrm{H}_{2} \mathrm{O} \\
\end{array}$ & $\begin{array}{c}\mathrm{C}_{6} \mathrm{H}_{5} \mathrm{OH}- \\
\left(\mathrm{H}_{2} \mathrm{O}\right)_{2} \\
\end{array}$ & $\begin{array}{c}\mathrm{C}_{6} \mathrm{H}_{5} \mathrm{OH}- \\
\left(\mathrm{H}_{2} \mathrm{O}\right)_{3} \\
\end{array}$ & $\begin{array}{c}\mathrm{C}_{6} \mathrm{H}_{5} \mathrm{OH}- \\
\mathrm{H}_{2} \mathrm{O} \\
\end{array}$ & $\begin{array}{c}\mathrm{C}_{6} \mathrm{H}_{5} \mathrm{OH}- \\
\left(\mathrm{H}_{2} \mathrm{O}\right)_{2} \\
\end{array}$ & $\begin{array}{c}\mathrm{C}_{6} \mathrm{H}_{5} \mathrm{OH}- \\
\left(\mathrm{H}_{2} \mathrm{O}\right)_{3} \\
\end{array}$ \\
\hline PBE & -1.92 & -1.86 & -1.75 & -0.56 & -0.51 & -0.61 & -2.49 & -2.37 & -2.36 \\
\hline TPSS & -1.93 & -1.87 & -1.75 & -0.55 & -0.50 & -0.60 & -2.48 & -2.36 & -2.35 \\
\hline M06-L & -1.94 & -1.87 & -1.77 & -0.52 & -0.47 & -0.56 & -2.46 & -2.34 & -2.33 \\
\hline B3LYP & -1.95 & -1.88 & -1.77 & -0.55 & -0.50 & -0.60 & -2.50 & -2.38 & -2.37 \\
\hline PBE0 & -1.91 & -1.85 & -1.74 & -0.58 & -0.52 & -0.62 & -2.49 & -2.37 & -2.36 \\
\hline TPSSh & -1.92 & -1.86 & -1.75 & -0.56 & -0.50 & -0.60 & -2.48 & -2.36 & -2.35 \\
\hline M06 & -1.95 & -1.87 & -1.77 & -0.54 & -0.49 & -0.58 & -2.48 & -2.36 & -2.35 \\
\hline M06-2X & -1.93 & -1.86 & -1.75 & -0.58 & -0.52 & -0.62 & -2.50 & -2.38 & -2.37 \\
\hline B2PLYP & -1.95 & -1.86 & -1.78 & -0.55 & -0.52 & -0.61 & -2.50 & -2.38 & -2.38 \\
\hline mPW2PLYP & -1.95 & -1.86 & -1.78 & -0.56 & -0.52 & -0.61 & -2.50 & -2.38 & -2.38 \\
\hline
\end{tabular}




\begin{tabular}{lcccc}
\hline & $\mathrm{X}$ & $\mathrm{Y}$ & $\mathrm{Z}$ & $\mathrm{N}$ \\
\cline { 1 - 1 } & \multicolumn{2}{c}{ AnilinelRed.Igas-phase } & & $\mathrm{C}$ \\
$\mathrm{C}$ & -0.07998 & -0.05882 & -0.25175 & $\mathrm{H}$ \\
$\mathrm{N}$ & -0.05754 & -0.08642 & -1.65493 & $\mathrm{C}$ \\
$\mathrm{C}$ & -0.08327 & 1.16346 & 0.44021 & $\mathrm{H}$ \\
$\mathrm{H}$ & -0.12309 & 2.09590 & -0.11931 & $\mathrm{C}$ \\
$\mathrm{C}$ & -0.03218 & 1.18694 & 1.83166 & $\mathrm{C}$ \\
$\mathrm{H}$ & -0.03811 & 2.14451 & 2.34611 & $\mathrm{C}$ \\
$\mathrm{C}$ & 0.02417 & 0.00003 & 2.56465 & $\mathrm{H}$ \\
$\mathrm{H}$ & 0.06306 & 0.02261 & 3.64945 & $\mathrm{C}$ \\
$\mathrm{C}$ & 0.02731 & -1.21657 & 1.87966 & $\mathrm{H}$ \\
$\mathrm{H}$ & 0.06820 & -2.15189 & 2.43207 & $\mathrm{H}$ \\
$\mathrm{C}$ & -0.02355 & -1.25121 & 0.48848 & $\mathrm{H}$ \\
$\mathrm{H}$ & -0.01684 & -2.20598 & -0.03352 & $\mathrm{H}$ \\
$\mathrm{H}$ & -0.42801 & -0.93970 & -2.05922 & $\mathrm{H}$ \\
$\mathrm{H}$ & -0.46951 & 0.73045 & -2.09287 & $\mathrm{H}$
\end{tabular}

$\begin{array}{lrrr}\mathrm{N} & 0.24190 & -0.06584 & -1.68880 \\ \mathrm{C} & 0.18538 & 1.16779 & 0.41965 \\ \mathrm{H} & 0.23532 & 2.10778 & -0.12639 \\ \mathrm{C} & 0.08585 & 1.17814 & 1.80984 \\ \mathrm{H} & 0.06500 & 2.13481 & 2.32813 \\ \mathrm{C} & 0.01404 & -0.00527 & 2.55217 \\ \mathrm{C} & -0.09498 & 0.01266 & 4.05949 \\ \mathrm{C} & 0.04824 & -1.21203 & 1.83965 \\ \mathrm{H} & -0.00233 & -2.15541 & 2.38023 \\ \mathrm{C} & 0.14710 & -1.23909 & 0.45232 \\ \mathrm{H} & 0.16694 & -2.19331 & -0.07063 \\ \mathrm{H} & -0.10216 & 1.03916 & 4.43872 \\ \mathrm{H} & 0.74570 & -0.51256 & 4.52934 \\ \mathrm{H} & -1.01539 & -0.47694 & 4.40079 \\ \mathrm{H} & 0.66629 & 0.75666 & -2.10443 \\ \mathrm{H} & 0.64399 & -0.91129 & -2.07977\end{array}$

p-MethoxyanilinelRed.Igas-phase

N-MethylanilinelRed.Igas-phase

$\begin{array}{lrrrr}\mathrm{C} & 0.07727 & -0.04344 & -0.33671 & \mathrm{C} \\ \mathrm{N} & 0.02632 & -0.02957 & -1.74787 & \mathrm{~N} \\ \mathrm{C} & 0.07115 & 1.14529 & 0.39979 & \mathrm{C} \\ \mathrm{H} & 0.08709 & 2.10093 & -0.12020 & \mathrm{C} \\ \mathrm{C} & 0.04048 & 1.13336 & 1.79728 & \mathrm{C} \\ \mathrm{H} & 0.03914 & 2.07814 & 2.33013 & \mathrm{C} \\ \mathrm{C} & 0.01447 & -0.08203 & 2.48567 & \mathrm{C} \\ \mathrm{O} & -0.01553 & -0.20961 & 3.85940 & \mathrm{C} \\ \mathrm{C} & 0.02142 & -1.27819 & 1.75767 & \mathrm{H} \\ \mathrm{H} & 0.00380 & -2.22041 & 2.29743 & \mathrm{H} \\ \mathrm{C} & 0.05055 & -1.25809 & 0.36980 & \mathrm{H} \\ \mathrm{H} & 0.04935 & -2.19831 & -0.17771 & \mathrm{H} \\ \mathrm{C} & -0.03361 & 1.00213 & 4.61587 & \mathrm{H} \\ \mathrm{H} & -0.06358 & 0.69441 & 5.66219 & \mathrm{H} \\ \mathrm{H} & -0.92249 & 1.60319 & 4.38246 & \mathrm{H} \\ \mathrm{H} & 0.86988 & 1.59899 & 4.43295 & \mathrm{H} \\ \mathrm{H} & 0.43498 & 0.80625 & -2.15431 & \mathrm{H} \\ \mathrm{H} & 0.44061 & -0.85473 & -2.17017 & \end{array}$

$\mathrm{C}$

$\mathrm{N}$

C

$\mathrm{C}$

$\mathrm{C}$

$\mathrm{C}$

C

$\mathrm{C}$

$\mathrm{H}$

$\mathrm{H}$

$\mathrm{H}$

$\mathrm{H}$

$\mathrm{H}$

$\mathrm{H}$

$\mathrm{H}$

$\mathrm{H}$

$\mathrm{H}$

p-Methylaniline/Red.Igas-phase

C
$\mathrm{N}$

DimethylaminelRed.Igas-phase

$$
\begin{array}{rrr}
-0.20243 & 1.25823 & -0.64778 \\
0.97754 & 1.85704 & -2.36192
\end{array}
$$




\begin{tabular}{|c|c|c|c|c|c|c|c|}
\hline $\mathrm{C}$ & -0.03471 & 1.50930 & -2.08208 & $\mathrm{H}$ & -1.77579 & -0.04184 & -1.24422 \\
\hline $\mathrm{C}$ & 0.17195 & 2.42107 & 0.16221 & $\mathrm{H}$ & -0.37142 & 0.73559 & -2.01961 \\
\hline $\mathrm{H}$ & -0.25595 & 0.59417 & -2.63983 & & & & \\
\hline $\mathrm{H}$ & -0.53370 & 3.23585 & -0.03612 & \multicolumn{4}{|c|}{ PiperidineIRed.|gas-phase } \\
\hline $\mathrm{H}$ & -0.75032 & 2.27791 & -2.39556 & $\mathrm{C}$ & -4.80934 & 1.28730 & 0.15769 \\
\hline $\mathrm{H}$ & 0.38678 & 0.47023 & -0.38187 & $\mathrm{C}$ & -4.77998 & -0.19584 & -0.22258 \\
\hline $\mathrm{H}$ & 0.10072 & 2.16329 & 1.22333 & $\mathrm{C}$ & -3.49390 & -0.85933 & 0.29412 \\
\hline $\mathrm{H}$ & 1.19099 & 2.80074 & -0.04098 & $\mathrm{C}$ & -2.25457 & -0.07698 & -0.16759 \\
\hline & & & & $\mathrm{C}$ & -2.38110 & 1.40158 & 0.21057 \\
\hline \multicolumn{4}{|c|}{ DiethylaminelRed.|gas-phase } & $\mathrm{N}$ & -3.61154 & 1.95235 & -0.38068 \\
\hline $\mathrm{N}$ & -0.22575 & 1.96877 & -0.25638 & $\mathrm{H}$ & -4.87542 & 1.37465 & 1.26114 \\
\hline $\mathrm{C}$ & 0.29066 & 1.49565 & -1.54633 & $\mathrm{H}$ & -5.69328 & 1.77573 & -0.26732 \\
\hline $\mathrm{C}$ & 0.35542 & 1.36841 & 0.95581 & $\mathrm{H}$ & -1.52930 & 1.97169 & -0.17667 \\
\hline $\mathrm{C}$ & -0.37227 & 0.13882 & 1.52285 & $\mathrm{H}$ & -2.37153 & 1.49253 & 1.31565 \\
\hline $\mathrm{C}$ & 0.06306 & 0.01968 & -1.92286 & $\mathrm{H}$ & -4.82563 & -0.27872 & -1.31585 \\
\hline $\mathrm{H}$ & 0.38384 & 2.14106 & 1.73685 & $\mathrm{H}$ & -5.66374 & -0.70066 & 0.18674 \\
\hline $\mathrm{H}$ & -0.15979 & 2.13222 & -2.31813 & $\mathrm{H}$ & -3.43751 & -1.90158 & -0.04243 \\
\hline $\mathrm{H}$ & 0.10209 & -0.19323 & 2.45481 & $\mathrm{H}$ & -3.51684 & -0.88032 & 1.39352 \\
\hline $\mathrm{H}$ & -0.37331 & -0.70013 & 0.82081 & $\mathrm{H}$ & -2.15385 & -0.15299 & -1.25766 \\
\hline $\mathrm{H}$ & 0.43630 & -0.17379 & -2.93648 & $\mathrm{H}$ & -1.34594 & -0.49743 & 0.28081 \\
\hline $\mathrm{H}$ & 0.58671 & -0.65851 & -1.24115 & $\mathrm{H}$ & -3.66282 & 2.95117 & -0.18500 \\
\hline $\mathrm{H}$ & -1.24150 & 1.88787 & -0.23355 & & & & \\
\hline $\mathrm{H}$ & -1.41545 & 0.38656 & 1.75540 & \multicolumn{4}{|c|}{ Anisole|Red.Igas-phase } \\
\hline $\mathrm{H}$ & -1.00412 & -0.23136 & -1.90287 & $\mathrm{C}$ & -0.00001 & -0.05451 & -0.43590 \\
\hline $\mathrm{H}$ & 1.39919 & 1.11805 & 0.73012 & $\mathrm{C}$ & -0.00001 & 1.14233 & 0.27525 \\
\hline $\mathrm{H}$ & 1.36716 & 1.70921 & -1.55980 & $\mathrm{H}$ & -0.00001 & 2.09103 & -0.25522 \\
\hline & & & & $\mathrm{C}$ & -0.00001 & 1.14822 & 1.67373 \\
\hline \multicolumn{4}{|c|}{ Pyrrolidine|Red.|gas-phase } & $\mathrm{H}$ & -0.00001 & 2.09313 & 2.20599 \\
\hline $\mathrm{C}$ & -0.41455 & -2.33585 & -0.82659 & $\mathrm{C}$ & -0.00001 & -0.06838 & 2.36549 \\
\hline $\mathrm{C}$ & -0.72361 & -2.60654 & -2.31246 & $\mathrm{C}$ & -0.00001 & -1.27764 & 1.65559 \\
\hline $\mathrm{C}$ & -0.98204 & -1.19410 & -2.91763 & $\mathrm{H}$ & -0.00001 & -2.20972 & 2.21277 \\
\hline $\mathrm{C}$ & -0.79962 & -0.22906 & -1.72930 & $\mathrm{C}$ & -0.00001 & -1.26479 & 0.26591 \\
\hline $\mathrm{N}$ & 0.13161 & -0.96742 & -0.85786 & $\mathrm{H}$ & -0.00001 & -2.20761 & -0.27471 \\
\hline $\mathrm{H}$ & 0.17458 & -0.55961 & 0.07561 & $\mathrm{H}$ & -0.00001 & -0.04877 & -1.52172 \\
\hline $\mathrm{H}$ & 0.31733 & -3.03221 & -0.40513 & $\mathrm{O}$ & 0.00000 & -0.18305 & 3.73433 \\
\hline $\mathrm{H}$ & -1.34330 & -2.40795 & -0.23040 & $\mathrm{C}$ & 0.00000 & 1.03219 & 4.49149 \\
\hline $\mathrm{H}$ & -1.58185 & -3.27452 & -2.42856 & $\mathrm{H}$ & 0.00000 & 0.72291 & 5.53745 \\
\hline $\mathrm{H}$ & 0.13440 & -3.07825 & -2.79912 & $\mathrm{H}$ & -0.89692 & 1.62858 & 4.28200 \\
\hline $\mathrm{H}$ & -1.97906 & -1.10402 & -3.35815 & $\mathrm{H}$ & 0.89692 & 1.62857 & 4.28200 \\
\hline $\mathrm{H}$ & -0.25054 & -0.97547 & -3.70035 & & & & \\
\hline
\end{tabular}


IndolelRed.Igas-phase

$\mathrm{C}$
$\mathrm{C}$
$\mathrm{C}$
$\mathrm{C}$
$\mathrm{C}$
$\mathrm{C}$
$\mathrm{H}$
$\mathrm{H}$
$\mathrm{H}$
$\mathrm{C}$
$\mathrm{H}$
$\mathrm{C}$
$\mathrm{N}$
$\mathrm{H}$
$\mathrm{H}$
$\mathrm{H}$

$\mathrm{H}$

$\mathrm{H}$

C

$\mathrm{H}$

C

$\mathrm{N}$

$\mathrm{H}$

$\mathrm{H}$

C

$\mathrm{H}$

C

$\mathrm{H}$

C

O

$\mathrm{H}$

C

$\mathrm{H}$

C

$\mathrm{H}$

$\mathrm{O}$

C

$\mathrm{H}$

$\mathrm{H}$

$\mathrm{H}$

C

C

2.15315

$-0.68489$

0.00001

2.15363

0.72506

0.00000

0.96642

1.44511

0.00000

$-0.25523$

0.74838

0.00001

$-0.22890$

$-0.67635$

0.00001

$0.96407 \quad-1.40503$

0.00001

0.98003

2.53197

0.00000

3.09936

$-1.21870$

0.00001

3.10300

1.25347

0.00000

$-1.63531$

1.14188

0.00001

$-2.02611$

2.14977

0.00002

$-2.38444$

$-0.00741$

0.00000

$-1.54239$

$-1.10618$

0.00000

$-3.45681$

$-0.14319$

$-1.84321$

$-2.06896$

0.00000

0.96650

$-2.49211$

0.00000

0.00002

p-Methoxyphenol|Red.Igas-phase

$\begin{array}{lll}0.04253 & 0.23653 & -0.17820\end{array}$

0.09863

1.35655

0.65976

0.17371

2.34431

0.21463

0.06178

1.20126

2.04164

0.10748

2.0782

2.68620

$-0.03359$

$-0.07658$

2.60438

$-0.09417$

$-0.28935$

3.96852

$-0.08705$

0.57342

4.41375

$-0.07133$

$-1.19365$

1.77368

$-0.13577$

$-2.18277$

2.21611

$-0.03364$

$-1.04123$

0.38621

$-0.06609 \quad-1.92586$

$-0.23963$

0.07154

0.49431

$-1.53193$

0.00622

$-0.63756$

$-2.40341$

0.03315

$-0.22983$

$-3.41453$

$\begin{array}{lll}0.86366 & -1.30630 & -2.25210\end{array}$

$-0.92575$

$-1.19853$

$-2.25567$

p-MethylphenollRed.lgas-phase

C

$$
-0.00002
$$

0.06307

$-0.20146$

$-0.00002$

1.28060

0.48219
C

$\mathrm{O}$

C

C

$\mathrm{H}$

C

C

$\mathrm{H}$

$\mathrm{H}$

$\mathrm{H}$

$\mathrm{H}$

$\mathrm{H}$

$\mathrm{H}$

$\mathrm{H}$

H

C

$\mathrm{H}$

$\mathrm{O}$

C

C

C

C

C

$\mathrm{H}$

$\mathrm{H}$

$\mathrm{H}$

$\mathrm{H}$

$\mathrm{H}$

\begin{tabular}{lrrr}
\multicolumn{4}{c}{ p-CyanophenollRed.Igas-phase } \\
C & 0.01268 & -0.00947 & -0.23662 \\
C & 0.00155 & 1.20396 & 0.43899 \\
C & -0.00469 & 1.22397 & 1.83930 \\
O & -0.01538 & 2.38710 & 2.56018 \\
C & 0.00004 & 0.02466 & 2.56215 \\
C & 0.01132 & -1.18562 & 1.88599 \\
H & 0.01517 & -2.11761 & 2.44179 \\
C & 0.01762 & -1.21873 & 0.47868 \\
C & 0.02936 & -2.46765 & -0.21534
\end{tabular}

$\begin{array}{rrr}-0.00001 & 1.29199 & 1.87772 \\ 0.00001 & 2.45700 & 2.61529 \\ -0.00002 & 0.08391 & 2.58112 \\ -0.00002 & -1.11854 & 1.88193 \\ -0.00001 & -2.05106 & 2.44274 \\ -0.00002 & -1.15753 & 0.47924 \\ 0.00000 & -2.47362 & -0.26402 \\ -0.00002 & 0.06979 & -1.28932 \\ 0.00001 & 3.20904 & 2.00064 \\ -0.00001 & 0.10311 & 3.66680 \\ -0.00002 & 2.21808 & -0.07192 \\ 0.00000 & -2.31264 & -1.34625 \\ -0.88305 & -3.07373 & -0.01331 \\ 0.88306 & -3.07371 & -0.01331\end{array}$

PhenollRed.Igas-phase

$\begin{array}{rrr}-0.14442 & -0.49232 & 0.00006 \\ -2.06519 & -0.49038 & 0.00014 \\ -1.35372 & -1.15173 & 0.00013 \\ 1.01301 & -1.27614 & 0.00004 \\ 2.25999 & -0.65588 & -0.00002 \\ 2.36644 & 0.73728 & -0.00007 \\ 1.20571 & 1.51049 & -0.00005 \\ -0.05020 & 0.90271 & 0.00002 \\ 0.91921 & -2.35777 & 0.00008 \\ 3.15722 & -1.26904 & -0.00003 \\ 3.34224 & 1.21333 & -0.00012 \\ 1.27159 & 2.59519 & -0.00008 \\ -0.95448 & 1.50933 & 0.00003\end{array}$

C 


$\begin{array}{lrrrr}\mathrm{H} & 0.01772 & -0.02758 & -1.32159 & \mathrm{H} \\ \mathrm{H} & -0.01760 & 3.14133 & 1.94709 & \mathrm{~S} \\ \mathrm{H} & -0.00505 & 0.06062 & 3.64670 & \mathrm{C} \\ \mathrm{H} & -0.00216 & 2.13739 & -0.12010 & \mathrm{H} \\ \mathrm{N} & 0.03363 & -3.48604 & -0.77826 & \mathrm{H} \\ & & & & \mathrm{H}\end{array}$

$\begin{array}{llll}\mathrm{H} & -0.00057 & 0.02577 & -1.60503 \\ \mathrm{~S} & 0.00113 & -0.45737 & 4.04233 \\ \mathrm{C} & -0.00051 & 1.23916 & 4.69731 \\ \mathrm{H} & 0.00023 & 1.12562 & 5.78384 \\ \mathrm{H} & -0.90011 & 1.77999 & 4.39323 \\ \mathrm{H} & 0.89745 & 1.78213 & 4.39222\end{array}$

DimethylsulfidelRed.Igas-phase

$\mathrm{S}$

C

C

$\mathrm{H}$

$\mathrm{H}$

$\mathrm{H}$

H

$\mathrm{H}$

H

$S$

C

C

$\mathrm{H}$

$\mathrm{H}$

S
C
C
H
H
H
H
H
H

S
C
C
H
H
H
H
H
H

H

H

C

C

$\mathrm{H}$

C

$\mathrm{H}$

C

C

$\mathrm{H}$

C

$\mathrm{H}$

$\begin{array}{rrr}-0.22551 & 0.17907 & 0.56222 \\ 0.21796 & 1.41051 & -0.70711 \\ 0.22183 & 1.11786 & 2.05970 \\ -0.00750 & 0.95715 & -1.67530 \\ -0.37630 & 2.32141 & -0.59089 \\ -0.37190 & 2.03313 & 2.13820 \\ -0.00186 & 0.47204 & 2.91216 \\ 1.28854 & 1.35983 & 2.06377 \\ 1.28459 & 1.64875 & -0.66279\end{array}$

DimethyldisulfidelRed.|gas-phase

(

$\mathrm{C}$
$\mathrm{C}$
$\mathrm{H}$
$\mathrm{C}$
$\mathrm{H}$
$\mathrm{C}$
$\mathrm{C}$
$\mathrm{H}$
$\mathrm{C}$
$\mathrm{H}$

$-0.24634$

$-0.89334$

$-0.99532$

1.29543

$-0.04995$

1.40902

$-0.26759$

$-1.90686$

$-0.12735$

$-0.92972$

0.49841

2.29266

$-1.16470$

$-0.35776$

1.42564

$-1.65566$

1.00909

2.51880

$-1.25276$

$-2.74355$

1.06437

$-1.40843$

ThioanisolelRed.|gas-phase

$-0.00022 \quad-0.04344$

$-0.52124$

0.00041

1.11094

0.25908

$0.00057 \quad 2.08930$

$-0.21460$

0.00085

1.03103

1.65335

0.00133

1.94510

2.23726

2.28308

1.49544

0.00005

$-0.21991$

$-1.38369$

1.97669

$-0.00009 \quad-2.35844$

$-0.00041 \quad-1.29169$

0.10761

$-0.00090$

$-2.20200$

$-0.48612$

$\mathrm{C}$
$\mathrm{C}$
$\mathrm{C}$
$\mathrm{C}$
$\mathrm{C}$
$\mathrm{H}$
$\mathrm{C}$
$\mathrm{H}$

C

C

C

C

C

$\mathrm{H}$

C

$\mathrm{H}$

H

H

Cl

N

H

H

C

C

C

O

C

C

H

C

H

H

H

Cl

C
p-ChloroanilinelRed.Igas-phase

$\begin{array}{lll}0.00128 & -0.09563 & -0.23869 \\ 0.11718 & 1.10464 & 0.45681 \\ 0.17386 & 1.12311 & 1.85998 \\ 0.10894 & -0.09994 & 2.54714 \\ -0.00740 & -1.30314 & 1.85667 \\ -0.05417 & -2.24208 & 2.39806 \\ -0.06127 & -1.29607 & 0.46493 \\ -0.03898 & -0.09790 & -1.32279 \\ 0.14689 & -0.11048 & 3.63409 \\ 0.16113 & 2.03976 & -0.09690 \\ -0.20546 & -2.81635 & -0.41173 \\ 0.21613 & 2.33739 & 2.55948 \\ 0.61448 & 2.27146 & 3.48974 \\ 0.62819 & 3.10569 & 2.04147\end{array}$

p-ChlorophenollRed.lgas-phase

$-0.00001 \quad 0.02144$

$-0.17985$

$\begin{array}{lll}0.00000 & 1.23268 & 0.51169\end{array}$

$\begin{array}{llll}\mathrm{H} & 0.00001 & 0.05747 & 3.70215\end{array}$

0.00001

1.24289

1.90956

$0.00000 \quad 2.40545$

2.64490

$0.00001 \quad 0.03706$

2.61693

$0.00000 \quad-1.17244 \quad 1.92828$

$\begin{array}{lll}0.00000 & -2.11135 & 2.47136\end{array}$

$\begin{array}{lll}-0.00001 & -1.17331 & 0.53388\end{array}$

$\begin{array}{lll}-0.00002 & 0.00928 & -1.26440\end{array}$

$\begin{array}{lll}-0.00001 & 3.16102 & 2.03450\end{array}$

0.00000

2.16899

$-0.04315$

$-0.00002$

$-2.70376$

$-0.33371$

AnilinelOx.lgas-phase

$-0.00001 \quad 0.00000$

$-0.22905$ 


$\begin{array}{lrrrrrrr}\mathrm{N} & 0.00001 & 0.00000 & -1.56902 & \mathrm{H} & 0.00000 & 2.17385 & 2.32702 \\ \mathrm{C} & -0.00002 & 1.24411 & 0.48347 & \mathrm{C} & 0.00000 & 0.01456 & 2.51581 \\ \mathrm{H} & -0.00002 & 2.17829 & -0.07129 & \mathrm{C} & -0.00001 & 0.00705 & 4.00728 \\ \mathrm{C} & 0.00000 & 1.23016 & 1.85687 & \mathrm{C} & 0.00001 & -1.21409 & 1.79220 \\ \mathrm{H} & 0.00001 & 2.16345 & 2.41035 & \mathrm{H} & 0.00000 & -2.15052 & 2.34265 \\ \mathrm{C} & 0.00001 & 0.00000 & 2.55454 & \mathrm{C} & 0.00001 & -1.23438 & 0.42280 \\ \mathrm{H} & 0.00002 & 0.00000 & 3.64038 & \mathrm{H} & 0.00001 & -2.17383 & -0.12307 \\ \mathrm{C} & -0.00001 & -1.23016 & 1.85687 & \mathrm{H} & -0.00001 & 0.85949 & -2.17488 \\ \mathrm{H} & 0.00000 & -2.16345 & 2.41035 & \mathrm{H} & -0.00001 & -0.87211 & -2.16820 \\ \mathrm{C} & -0.00002 & -1.24411 & 0.48347 & \mathrm{H} & -0.00001 & 1.01469 & 4.42519 \\ \mathrm{H} & -0.00001 & -2.17829 & -0.07129 & \mathrm{H} & 0.87735 & -0.53629 & 4.38466 \\ \mathrm{H} & 0.00002 & 0.86644 & -2.09961 & \mathrm{H} & -0.87736 & -0.53629 & 4.38465 \\ \mathrm{H} & 0.00002 & -0.86644 & -2.09960 & & & & \end{array}$

N-MethylanilinelOx.|gas-phase

\begin{tabular}{|c|c|c|c|c|c|c|c|}
\hline $\mathrm{C}$ & 0.00000 & -0.02809 & -0.33711 & $\mathrm{~N}$ & -1.41635 & -0.09948 & -0.10588 \\
\hline $\mathrm{N}$ & -0.00003 & -0.00977 & -1.68265 & $\mathrm{C}$ & -2.08805 & -0.66721 & -1.12734 \\
\hline $\mathrm{C}$ & 0.00003 & 1.19677 & 0.39724 & $\mathrm{C}$ & -1.40343 & -1.25107 & -2.23689 \\
\hline $\mathrm{H}$ & 0.00002 & 2.14077 & -0.14049 & $\mathrm{C}$ & -2.13756 & -1.81809 & -3.25407 \\
\hline $\mathrm{C}$ & 0.00005 & 1.18670 & 1.76963 & $\mathrm{C}$ & -3.54863 & -1.82333 & -3.20278 \\
\hline $\mathrm{H}$ & 0.00006 & 2.12306 & 2.31522 & $\mathrm{C}$ & -4.23076 & -1.24889 & -2.11091 \\
\hline $\mathrm{C}$ & 0.00006 & -0.05446 & 2.46104 & $\mathrm{C}$ & -3.52016 & -0.67606 & -1.08201 \\
\hline $\mathrm{O}$ & 0.00004 & -0.18470 & 3.78343 & $\mathrm{H}$ & 0.48956 & -0.98820 & 0.04907 \\
\hline $\mathrm{C}$ & 0.00002 & -1.28103 & 1.73388 & $\mathrm{H}$ & 0.45290 & 0.58488 & -0.80164 \\
\hline $\mathrm{H}$ & 0.00001 & -2.21051 & 2.29366 & $\mathrm{H}$ & 0.25394 & 0.52088 & 0.96904 \\
\hline $\mathrm{C}$ & 0.00000 & -1.27110 & 0.36756 & $\mathrm{H}$ & -1.97860 & 0.29391 & 0.64559 \\
\hline $\mathrm{H}$ & -0.00003 & -2.20368 & -0.18929 & $\mathrm{H}$ & -0.31970 & -1.24564 & -2.27371 \\
\hline $\mathrm{H}$ & -0.00003 & 0.86148 & -2.20029 & $\mathrm{H}$ & -1.62918 & -2.26480 & -4.10236 \\
\hline $\mathrm{H}$ & -0.00005 & -0.86787 & -2.22198 & $\mathrm{H}$ & -4.11309 & -2.27466 & -4.01300 \\
\hline $\mathrm{C}$ & -0.00003 & 0.99927 & 4.63775 & $\mathrm{H}$ & -5.31534 & -1.25910 & -2.08305 \\
\hline $\mathrm{H}$ & -0.00004 & 0.60341 & 5.65083 & $\mathrm{H}$ & -4.02856 & -0.22826 & -0.23180 \\
\hline $\mathrm{H}$ & -0.90325 & 1.58608 & 4.45318 & & & & \\
\hline $\mathrm{H}$ & 0.90316 & 1.58615 & 4.45322 & \multicolumn{4}{|c|}{ DimethylaminelOx.|gas-phase } \\
\hline & \multirow{2}{*}{\multicolumn{3}{|c|}{ p-MethylanilinelOx.|gas-phase }} & $\mathrm{N}$ & -0.01818 & 1.37967 & -0.71064 \\
\hline & & & & $\mathrm{H}$ & -0.02165 & 2.55235 & -2.43644 \\
\hline $\mathrm{C}$ & 0.00000 & 0.00087 & -0.30039 & $\mathrm{C}$ & -0.20237 & 1.52327 & -2.13138 \\
\hline $\mathrm{N}$ & -0.00001 & -0.00427 & -1.64185 & $\mathrm{C}$ & 0.35135 & 2.42880 & 0.20359 \\
\hline $\mathrm{C}$ & 0.00001 & 1.24143 & 0.41232 & $\mathrm{H}$ & 0.47917 & 0.82851 & -2.64845 \\
\hline $\mathrm{H}$ & 0.00000 & 2.17649 & -0.14110 & $\mathrm{H}$ & 0.47831 & 3.36986 & -0.32839 \\
\hline & 0.00001 & 1.23337 & 1.78437 & $\mathrm{H}$ & -1.22526 & 1.20440 & -2.38944 \\
\hline
\end{tabular}




\begin{tabular}{|c|c|c|c|c|c|c|c|}
\hline $\mathrm{H}$ & -0.16327 & 0.44582 & -0.31413 & $\mathrm{C}$ & -4.78387 & -0.17410 & -0.22380 \\
\hline $\mathrm{H}$ & -0.42624 & 2.51139 & 0.98021 & $\mathrm{C}$ & -3.49591 & -0.83558 & 0.25951 \\
\hline $\mathrm{H}$ & 1.27832 & 2.13553 & 0.72249 & $\mathrm{C}$ & -2.25395 & -0.05790 & -0.16781 \\
\hline & & & & $\mathrm{C}$ & -2.36269 & 1.43888 & 0.34320 \\
\hline & Diethylamine & Igas-phase & & $\mathrm{N}$ & -3.61537 & 1.95519 & -0.12916 \\
\hline $\mathrm{N}$ & -0.30711 & 1.45143 & -0.26665 & $\mathrm{H}$ & -4.87261 & 1.31445 & 1.38234 \\
\hline $\mathrm{C}$ & 0.29543 & 1.49067 & -1.57734 & $\mathrm{H}$ & -5.69327 & 1.85631 & -0.12571 \\
\hline $\mathrm{C}$ & 0.39363 & 1.24634 & 0.98281 & $\mathrm{H}$ & -1.53933 & 2.04693 & -0.03313 \\
\hline $\mathrm{C}$ & -0.35670 & 0.29912 & 1.93152 & $\mathrm{H}$ & -2.37308 & 1.42892 & 1.43798 \\
\hline $\mathrm{C}$ & 0.04591 & 0.14986 & -2.33707 & $\mathrm{H}$ & -4.85046 & -0.16992 & -1.31646 \\
\hline $\mathrm{H}$ & 0.47225 & 2.24547 & 1.45430 & $\mathrm{H}$ & -5.68177 & -0.65803 & 0.17300 \\
\hline $\mathrm{H}$ & -0.17691 & 2.30652 & -2.13777 & $\mathrm{H}$ & -3.44003 & -1.84572 & -0.17030 \\
\hline $\mathrm{H}$ & 0.20398 & 0.24532 & 2.86791 & $\mathrm{H}$ & -3.51454 & -0.95497 & 1.34927 \\
\hline $\mathrm{H}$ & -0.42728 & -0.70367 & 1.50373 & $\mathrm{H}$ & -2.13997 & -0.04535 & -1.25649 \\
\hline $\mathrm{H}$ & 0.50335 & 0.26168 & -3.32387 & $\mathrm{H}$ & -1.33384 & -0.45855 & 0.26898 \\
\hline $\mathrm{H}$ & 0.51581 & -0.68343 & -1.81120 & $\mathrm{H}$ & -3.62040 & 2.49704 & -0.99513 \\
\hline $\mathrm{H}$ & -1.32701 & 1.53468 & -0.21580 & & & & \\
\hline $\mathrm{H}$ & -1.36005 & 0.67196 & 2.15846 & \multicolumn{4}{|c|}{ AnisolelOx.Igas-phase } \\
\hline $\mathrm{H}$ & -1.02272 & -0.03990 & -2.46004 & $\mathrm{C}$ & -0.00002 & -0.07133 & -0.42645 \\
\hline $\mathrm{H}$ & 1.40933 & 0.91614 & 0.74897 & $\mathrm{C}$ & -0.00001 & 1.17063 & 0.26593 \\
\hline \multirow[t]{3}{*}{$\mathrm{H}$} & 1.36448 & 1.67651 & -1.45929 & $\mathrm{H}$ & -0.00001 & 2.09627 & -0.30088 \\
\hline & & & & $\mathrm{C}$ & 0.00000 & 1.20498 & 1.63914 \\
\hline & \multicolumn{3}{|c|}{ PyrrolidinelOx.lgas-phase } & $\mathrm{H}$ & 0.00000 & 2.14767 & 2.17412 \\
\hline $\mathrm{C}$ & -0.25138 & -2.38508 & -0.90049 & $\mathrm{C}$ & 0.00000 & -0.03534 & 2.34740 \\
\hline $\mathrm{C}$ & -0.95674 & -2.58968 & -2.25145 & $\mathrm{C}$ & 0.00000 & -1.29192 & 1.64898 \\
\hline $\mathrm{C}$ & -0.80171 & -1.21985 & -2.94775 & $\mathrm{H}$ & 0.00000 & -2.20334 & 2.23840 \\
\hline $\mathrm{C}$ & -0.89216 & -0.21662 & -1.78595 & $\mathrm{C}$ & -0.00001 & -1.29691 & 0.27559 \\
\hline $\mathrm{N}$ & -0.40345 & -0.96782 & -0.64929 & $\mathrm{H}$ & -0.00001 & -2.23463 & -0.27009 \\
\hline $\mathrm{H}$ & -0.18559 & -0.53505 & 0.25239 & $\mathrm{H}$ & -0.00002 & -0.07283 & -1.51260 \\
\hline $\mathrm{H}$ & 0.83699 & -2.58585 & -0.96022 & $\mathrm{O}$ & 0.00000 & -0.14562 & 3.66068 \\
\hline $\mathrm{H}$ & -0.62350 & -2.96082 & -0.04326 & $\mathrm{C}$ & 0.00000 & 1.03786 & 4.53123 \\
\hline $\mathrm{H}$ & -2.01118 & -2.82734 & -2.08070 & $\mathrm{H}$ & -0.00001 & 0.62730 & 5.53791 \\
\hline $\mathrm{H}$ & -0.50760 & -3.40546 & -2.81858 & $\mathrm{H}$ & -0.90462 & 1.61986 & 4.34254 \\
\hline $\mathrm{H}$ & -1.57312 & -1.03671 & -3.69627 & $\mathrm{H}$ & 0.90462 & 1.61985 & 4.34255 \\
\hline $\mathrm{H}$ & 0.17613 & -1.13226 & -3.43110 & & & & \\
\hline $\mathrm{H}$ & -1.93825 & 0.06881 & -1.55681 & \multicolumn{4}{|c|}{ IndolelOx.|gas-phase } \\
\hline \multirow[t]{3}{*}{$\mathrm{H}$} & -0.33230 & 0.72247 & -1.88426 & $\mathrm{C}$ & 2.15482 & -0.66518 & 0.00000 \\
\hline & & & & $\mathrm{C}$ & 2.16835 & 0.73019 & 0.00002 \\
\hline & \multicolumn{3}{|c|}{ PiperidinelOx.|gas-phase } & $\mathrm{C}$ & 0.97371 & 1.45600 & 0.00000 \\
\hline & -4.83521 & 1.32556 & 0.28817 & $\mathrm{C}$ & -0.25110 & 0.74452 & 0.00000 \\
\hline
\end{tabular}




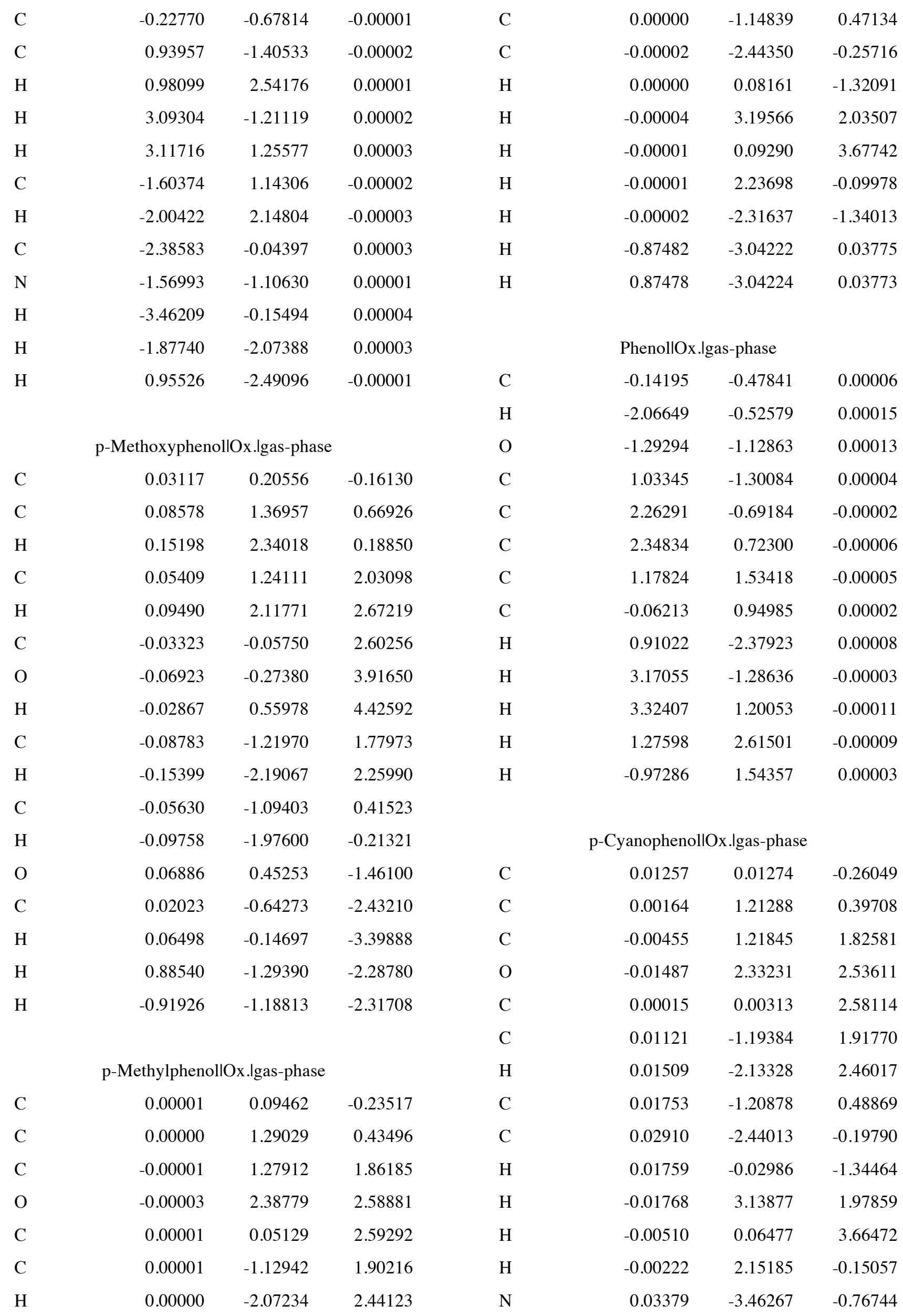


DimethylsulfidelOx.|gas-phase$$
\text { S }
$$

$\mathrm{C}$

C

$-0.21522$

0.23227

0.56818

0.21303

1.39260

$-0.73163$

0.21419

1.09774

2.08011

$-0.02555$

0.91267

$-1.68214$

$-0.37109$

2.31117

$-0.59196$

$-0.36580$

2.02794

2.13208

$-0.02826$

0.43295

2.91077

1.28521

1.33600

2.05974

1.28290

1.62591

$-0.66003$

DimethyldisulfidelOx.|gas-phase
S

S

C

C

$\mathrm{H}$

$\mathrm{H}$

$\mathrm{H}$

$\mathrm{H}$

$\mathrm{H}$

$\mathrm{H}$

C

C

$\mathrm{H}$

C

$\mathrm{H}$

C

C

$\mathrm{H}$

C

$\mathrm{H}$

$\mathrm{H}$

S

C

$\mathrm{H}$

$\mathrm{H}$
0.16688

0.43509

$-0.18771$

0.00896

0.14827

$-0.16892$

1.62054

$-1.17649$

$-0.54808$

2.36801

$-0.03273$

1.43562

0.52717

$-1.92400$

0.01281

$-0.99174$

1.18951

1.90054

$-1.21025$

$-1.45643$

ThioanisolelOx.|gas-phase

$0.00000 \quad-0.06034$

$-0.00001$

1.13250

$-0.00001$

2.09168

$-0.00001$

1.08468

$-0.00001$

0.00000

2.00093

$-0.18037$

$-0.00001$

$-1.38667$

$-1.07670$

0.86498

1.65380

$-1.86557$

1.26962

2.71606

$-1.48097$

$-2.92773$

1.48452

$-1.69679$

$-2.34598$

$-0.00001$

$-1.31533$

$-0.00002$

$-2.22503$

$-0.00001$

$-0.00529$

$-0.51264$

0.24247

$-0.26537$

1.62140

2.20033

2.26499

1.49906

2.00890

0.12103

$-0.47032$

$-1.59726$

0.00000

$-0.41978$

3.96704

0.00000

1.22626

4.72743

0.00000

1.03623

5.80255

$-0.90383$

1.76951

4.44242
$-0.00001$
C

C

C

C

C

$\mathrm{H}$

C

$\mathrm{H}$

$\mathrm{H}$

$\mathrm{H}$

$\mathrm{Cl}$

$\mathrm{N}$

$\mathrm{H}$

$\mathrm{H}$

C

C

C

$\mathrm{O}$

C

C

$\mathrm{H}$

C

$\mathrm{H}$

$\mathrm{H}$

$\mathrm{H}$

$\mathrm{H}$

$\mathrm{Cl}$

Cl

C

N

C

$\mathrm{H}$

C

$\mathrm{H}$
p-ChloroanilinelOx.Igas-phase

$\begin{array}{rrr}-0.00001 & -0.02293 & -0.23096 \\ 0.00000 & 1.16877 & 0.44668 \\ 0.00000 & 1.17588 & 1.87777 \\ 0.00001 & -0.06110 & 2.59746 \\ 0.00000 & -1.24952 & 1.91406 \\ 0.00000 & -2.19651 & 2.44245 \\ -0.00001 & -1.23921 & 0.49675 \\ -0.00002 & -0.04783 & -1.31510 \\ 0.00002 & -0.05047 & 3.68374 \\ 0.00000 & 2.11037 & -0.09508 \\ -0.00003 & -2.72050 & -0.35026 \\ -0.00001 & 2.34040 & 2.54363 \\ -0.00001 & 3.23025 & 2.05551 \\ -0.00001 & 2.37113 & 3.55809\end{array}$

p-ChlorophenollOx.Igas-phase

$\begin{array}{rrr}-0.00001 & 0.04562 & -0.20190 \\ -0.00001 & 1.24061 & 0.46956 \\ 0.00000 & 1.23614 & 1.89569 \\ 0.00000 & 2.34787 & 2.61827 \\ 0.00000 & 0.01450 & 2.63511 \\ 0.00000 & -1.17783 & 1.96067 \\ 0.00000 & -2.12394 & 2.49083 \\ -0.00001 & -1.17015 & 0.53938 \\ -0.00001 & 0.01009 & -1.28596 \\ 0.00000 & 3.15500 & 2.06378 \\ 0.00001 & 0.06180 & 3.71937 \\ -0.00001 & 2.18291 & -0.07239 \\ -0.00001 & -2.64719 & -0.30028\end{array}$

AnilinelRed.ISMD

$\begin{array}{lll}-0.00008 & 0.00004 & -0.24285\end{array}$

$\begin{array}{lll}-0.00036 & -0.00002 & -1.61655\end{array}$

$\begin{array}{lll}-0.00004 & 1.21166 & 0.48157\end{array}$

$\begin{array}{lll}0.00001 & 2.15512 & -0.05963\end{array}$

$\begin{array}{lll}0.00010 & 1.20298 & 1.87430\end{array}$

$\begin{array}{lll}0.00021 & 2.15077 & 2.40701\end{array}$ 


$\begin{array}{lllll}\mathrm{C} & 0.00001 & -0.00001 & 2.58862 & \mathrm{C} \\ \mathrm{H} & 0.00000 & -0.00002 & 3.67444 & \mathrm{H} \\ \mathrm{C} & -0.00008 & -1.20298 & 1.87428 & \mathrm{H} \\ \mathrm{H} & -0.00012 & -2.15082 & 2.40691 & \mathrm{H} \\ \mathrm{C} & 0.00001 & -1.21159 & 0.48156 & \mathrm{H} \\ \mathrm{H} & -0.00002 & -2.15502 & -0.05969 & \mathrm{H} \\ \mathrm{H} & -0.00038 & 0.86565 & -2.13397 & \mathrm{H} \\ \mathrm{H} & 0.00083 & -0.86580 & -2.13380 & \end{array}$

$\begin{array}{lrrr}\mathrm{C} & 0.00014 & -1.20539 & 0.40633 \\ \mathrm{H} & 0.00007 & -2.15195 & -0.12984 \\ \mathrm{H} & -0.00114 & 0.86329 & -2.21916 \\ \mathrm{H} & 0.00021 & -0.86805 & -2.21454 \\ \mathrm{H} & -0.00012 & 1.02049 & 4.43633 \\ \mathrm{H} & 0.88178 & -0.51438 & 4.44627 \\ \mathrm{H} & -0.88203 & -0.51436 & 4.44614\end{array}$

N-Methylaniline|Red.ISMD

p-MethoxyanilinelRed.ISMD

C

C

$\mathrm{H}$

$\mathrm{C}$

$\mathrm{H}$

$\mathrm{C}$

$\mathrm{N}$

$\mathrm{C}$

$\mathrm{H}$

$\mathrm{C}$

$\mathrm{H}$

$\mathrm{C}$

$\mathrm{H}$

$\mathrm{H}$

$\mathrm{H}$

$\mathrm{H}$

$\mathrm{H}$

$\begin{array}{lll}-0.00006 & 0.70208 & 5.67442\end{array}$

$\begin{array}{lll}-0.89726 & 1.59935 & 4.41637\end{array}$

$\begin{array}{lll}0.89812 & 1.59839 & 4.41643\end{array}$

p-MethylanilineIRed.ISMD

C

$\mathrm{N}$

C

$\mathrm{H}$

$\mathrm{C}$

$\mathrm{H}$

C

C

C

$\mathrm{H}$

$\mathrm{H}$

$\mathrm{C}$
$\mathrm{C}$
$\mathrm{C}$
$\mathrm{C}$
$\mathrm{C}$
$\mathrm{C}$

$0.89812-1.59839-4.41643$

$\mathrm{N}$

$\mathrm{H}$

C

C

$\mathrm{H}$

$\mathrm{H}$

$\mathrm{H}$

$\mathrm{H}$

$\mathrm{H}$

$\mathrm{H}$

$\begin{array}{rrr}0.00003 & -0.04521 & -0.36634 \\ -0.00012 & 1.15536 & 0.34760 \\ -0.00018 & 2.10297 & -0.18577 \\ -0.00024 & 1.17462 & 1.74278 \\ -0.00040 & 2.13115 & 2.25489 \\ -0.00016 & -0.03351 & 2.47669 \\ -0.00022 & -0.08910 & 3.85033 \\ 0.00020 & 1.03628 & 4.77247 \\ -0.00055 & -1.01252 & 4.26208 \\ 0.00001 & -1.24696 & 1.74400 \\ 0.00015 & -2.18910 & 2.28859 \\ 0.00012 & -1.24652 & 0.35455 \\ 0.00027 & -2.19755 & -0.17254 \\ 0.00053 & 1.97700 & 4.22397 \\ 0.88933 & 1.01756 & 5.41316 \\ -0.88892 & 1.01825 & 5.41318 \\ 0.00016 & -0.04760 & -1.45210\end{array}$

DimethylaminelRed.ISMD

$\begin{array}{ccc}-0.23154 & 1.23910 & -0.63667 \\ 0.97841 & 1.86069 & -2.31297 \\ -0.03872 & 1.51073 & -2.07458 \\ 0.16533 & 2.41945 & 0.15569 \\ -0.23745 & 0.60369 & -2.65144 \\ -0.53056 & 3.24096 & -0.04127 \\ -0.74563 & 2.28137 & -2.39754 \\ 0.42566 & 0.49589 & -0.39399 \\ 0.11771 & 2.18150 & 1.22158 \\ 1.18262 & 2.77182 & -0.07859\end{array}$

Diethylamine|Red.ISMD 


\begin{tabular}{|c|c|c|c|c|c|c|c|}
\hline $\mathrm{N}$ & -0.27093 & 1.97790 & -0.24942 & $\mathrm{H}$ & -4.85095 & 1.38601 & 1.24218 \\
\hline $\mathrm{C}$ & 0.26731 & 1.52789 & -1.55496 & $\mathrm{H}$ & -5.69929 & 1.76822 & -0.26624 \\
\hline $\mathrm{C}$ & 0.37348 & 1.32149 & 0.92338 & $\mathrm{H}$ & -1.52299 & 1.96507 & -0.17606 \\
\hline $\mathrm{C}$ & -0.42820 & 0.17016 & 1.52903 & $\mathrm{H}$ & -2.39582 & 1.50184 & 1.29521 \\
\hline $\mathrm{C}$ & 0.12861 & 0.03943 & -1.88813 & $\mathrm{H}$ & -4.83710 & -0.29936 & -1.31578 \\
\hline $\mathrm{H}$ & 0.53237 & 2.07922 & 1.70079 & $\mathrm{H}$ & -5.66238 & -0.69080 & 0.19696 \\
\hline $\mathrm{H}$ & -0.23869 & 2.11514 & -2.32916 & $\mathrm{H}$ & -3.43728 & -1.90266 & -0.04287 \\
\hline $\mathrm{H}$ & 0.08006 & -0.22342 & 2.41769 & $\mathrm{H}$ & -3.51664 & -0.87840 & 1.39170 \\
\hline $\mathrm{H}$ & -0.56458 & -0.65323 & 0.82072 & $\mathrm{H}$ & -2.14066 & -0.17239 & -1.25737 \\
\hline $\mathrm{H}$ & 0.52437 & -0.15539 & -2.89270 & $\mathrm{H}$ & -1.34849 & -0.48732 & 0.29052 \\
\hline $\mathrm{H}$ & 0.68521 & -0.59099 & -1.18636 & $\mathrm{H}$ & -3.66461 & 2.94756 & -0.10589 \\
\hline $\mathrm{H}$ & -1.26092 & 1.72546 & -0.23041 & & & & \\
\hline $\mathrm{H}$ & -1.42056 & 0.51757 & 1.84207 & \multicolumn{4}{|c|}{ AnisolelRed.ISMD } \\
\hline $\mathrm{H}$ & -0.92284 & -0.27114 & -1.87454 & $\mathrm{C}$ & -0.00003 & -0.05461 & -0.43755 \\
\hline $\mathrm{H}$ & 1.36777 & 0.97710 & 0.61957 & $\mathrm{C}$ & -0.00003 & 1.14220 & 0.27744 \\
\hline $\mathrm{H}$ & 1.32488 & 1.81325 & -1.58783 & $\mathrm{H}$ & -0.00003 & 2.09218 & -0.25001 \\
\hline & & & & $\mathrm{C}$ & -0.00004 & 1.14562 & 1.67671 \\
\hline \multicolumn{4}{|c|}{ PyrrolidineIRed.ISMD } & $\mathrm{H}$ & -0.00002 & 2.08881 & 2.21116 \\
\hline $\mathrm{C}$ & -0.40599 & -2.33379 & -0.83484 & $\mathrm{C}$ & -0.00005 & -0.07415 & 2.36306 \\
\hline $\mathrm{C}$ & -0.72198 & -2.60620 & -2.31371 & $\mathrm{C}$ & -0.00004 & -1.28346 & 1.65257 \\
\hline $\mathrm{C}$ & -0.98058 & -1.19468 & -2.91825 & $\mathrm{H}$ & -0.00001 & -2.22021 & 2.20250 \\
\hline $\mathrm{C}$ & -0.78803 & -0.23337 & -1.73489 & $\mathrm{C}$ & 0.00001 & -1.26779 & 0.26105 \\
\hline $\mathrm{N}$ & 0.16229 & -0.96104 & -0.85443 & $\mathrm{H}$ & 0.00007 & -2.20934 & -0.28109 \\
\hline $\mathrm{H}$ & 0.10832 & -0.56774 & 0.08641 & $\mathrm{H}$ & 0.00002 & -0.04617 & -1.52332 \\
\hline $\mathrm{H}$ & 0.30896 & -3.04156 & -0.40695 & $\mathrm{O}$ & -0.00006 & -0.18697 & 3.73974 \\
\hline $\mathrm{H}$ & -1.32912 & -2.37606 & -0.23618 & $\mathrm{C}$ & 0.00002 & 1.04382 & 4.49591 \\
\hline $\mathrm{H}$ & -1.58268 & -3.27172 & -2.42103 & $\mathrm{H}$ & 0.00002 & 0.73883 & 5.54237 \\
\hline $\mathrm{H}$ & 0.13049 & -3.08301 & -2.80659 & $\mathrm{H}$ & -0.89844 & 1.63192 & 4.28193 \\
\hline $\mathrm{H}$ & -1.98115 & -1.10060 & -3.34864 & $\mathrm{H}$ & 0.89853 & 1.63184 & 4.28197 \\
\hline $\mathrm{H}$ & -0.25666 & -0.97899 & -3.70970 & & & & \\
\hline $\mathrm{H}$ & -1.74975 & -0.05949 & -1.22776 & \multicolumn{4}{|c|}{ Indole|Red.ISMD } \\
\hline \multirow[t]{3}{*}{$\mathrm{H}$} & -0.37798 & 0.73698 & -2.02720 & $\mathrm{C}$ & 2.15314 & -0.68642 & 0.00000 \\
\hline & & & & $\mathrm{C}$ & 2.15608 & 0.72582 & 0.00001 \\
\hline & \multicolumn{3}{|c|}{ PiperidinelRed.ISMD } & $\mathrm{C}$ & 0.96852 & 1.44911 & 0.00000 \\
\hline $\mathrm{C}$ & -4.80610 & 1.28624 & 0.14309 & $\mathrm{C}$ & -0.25544 & 0.75235 & -0.00002 \\
\hline $\mathrm{C}$ & -4.77854 & -0.19646 & -0.22394 & $\mathrm{C}$ & -0.23158 & -0.67535 & 0.00000 \\
\hline $\mathrm{C}$ & -3.49370 & -0.86030 & 0.29270 & $\mathrm{C}$ & 0.96170 & -1.40568 & -0.00002 \\
\hline $\mathrm{C}$ & -2.25587 & -0.07754 & -0.16933 & $\mathrm{H}$ & 0.98466 & 2.53607 & 0.00002 \\
\hline $\mathrm{C}$ & -2.38371 & 1.40046 & 0.19542 & $\mathrm{H}$ & 3.09874 & -1.22140 & 0.00000 \\
\hline $\mathrm{N}$ & -3.61217 & 1.97299 & -0.40585 & $\mathrm{H}$ & 3.10631 & 1.25300 & 0.00007 \\
\hline
\end{tabular}




\begin{tabular}{|c|c|c|c|c|c|c|c|}
\hline $\mathrm{C}$ & -1.63659 & 1.14421 & -0.00003 & $\mathrm{H}$ & -0.00003 & 2.22247 & -0.06726 \\
\hline $\mathrm{H}$ & -2.03075 & 2.15152 & -0.00002 & $\mathrm{H}$ & 0.00002 & -2.31408 & -1.34639 \\
\hline $\mathrm{C}$ & -2.38138 & -0.01230 & -0.00001 & $\mathrm{H}$ & -0.88213 & -3.07476 & -0.01172 \\
\hline $\mathrm{N}$ & -1.54148 & -1.10695 & 0.00001 & $\mathrm{H}$ & 0.88193 & -3.07490 & -0.01165 \\
\hline $\mathrm{H}$ & -3.45287 & -0.15266 & 0.00011 & & & & \\
\hline $\mathrm{H}$ & -1.84469 & -2.07109 & 0.00006 & \multicolumn{4}{|c|}{ PhenollRed.ISMD } \\
\hline $\mathrm{H}$ & 0.95845 & -2.49207 & -0.00003 & $\mathrm{C}$ & 0.00946 & -0.01666 & -0.25930 \\
\hline & & & & $\mathrm{H}$ & 0.01551 & -0.03879 & -1.34480 \\
\hline \multicolumn{4}{|c|}{ p-MethoxyphenollRed.ISMD } & $\mathrm{C}$ & -0.00688 & 1.20432 & 0.42159 \\
\hline $\mathrm{C}$ & 0.03086 & 0.24167 & -0.17968 & $\mathrm{H}$ & -0.01356 & 2.13792 & -0.13398 \\
\hline $\mathrm{C}$ & 0.09256 & 1.36022 & 0.66059 & $\mathrm{C}$ & -0.01478 & 1.24202 & 1.81572 \\
\hline $\mathrm{H}$ & 0.16541 & 2.35199 & 0.22410 & $\mathrm{H}$ & -0.02741 & 2.18681 & 2.35120 \\
\hline $\mathrm{C}$ & 0.06095 & 1.20214 & 2.04322 & $\mathrm{C}$ & -0.00611 & 0.04229 & 2.53316 \\
\hline $\mathrm{H}$ & 0.10915 & 2.07399 & 2.69100 & $\mathrm{O}$ & -0.01438 & 0.12641 & 3.91791 \\
\hline $\mathrm{C}$ & -0.03273 & -0.07910 & 2.59663 & $\mathrm{H}$ & -0.00645 & -0.77661 & 4.28287 \\
\hline $\mathrm{O}$ & -0.06793 & -0.29141 & 3.97224 & $\mathrm{C}$ & 0.01038 & -1.18631 & 1.86641 \\
\hline $\mathrm{H}$ & -0.01577 & 0.57267 & 4.41763 & $\mathrm{H}$ & 0.01713 & -2.11267 & 2.43590 \\
\hline $\mathrm{C}$ & -0.09441 & -1.19459 & 1.76436 & $\mathrm{C}$ & 0.01801 & -1.20782 & 0.47043 \\
\hline $\mathrm{H}$ & -0.16718 & -2.18763 & 2.19780 & $\mathrm{H}$ & 0.03078 & -2.16398 & -0.04524 \\
\hline $\mathrm{C}$ & -0.06294 & -1.03854 & 0.37536 & & & & \\
\hline $\mathrm{H}$ & -0.11224 & -1.92050 & -0.25289 & \multicolumn{4}{|c|}{ p-CyanophenollRed.ISMD } \\
\hline $\mathrm{O}$ & 0.06932 & 0.50056 & -1.54177 & $\mathrm{C}$ & 0.01246 & -0.00575 & -0.24086 \\
\hline $\mathrm{C}$ & 0.00509 & -0.64479 & -2.41649 & $\mathrm{C}$ & 0.00160 & 1.20512 & 0.43503 \\
\hline $\mathrm{H}$ & 0.04716 & -0.23985 & -3.42780 & $\mathrm{C}$ & -0.00446 & 1.21633 & 1.83630 \\
\hline $\mathrm{H}$ & 0.85815 & -1.31165 & -2.25025 & $\mathrm{O}$ & -0.01504 & 2.38641 & 2.55402 \\
\hline $\mathrm{H}$ & -0.93401 & -1.19113 & -2.27640 & $\mathrm{C}$ & 0.00011 & 0.01882 & 2.56414 \\
\hline & & & & $\mathrm{C}$ & 0.01107 & -1.19142 & 1.89029 \\
\hline \multicolumn{4}{|c|}{ p-MethylphenollRed.ISMD } & $\mathrm{H}$ & 0.01482 & -2.12321 & 2.44502 \\
\hline $\mathrm{C}$ & 0.00005 & 0.06411 & -0.20246 & $\mathrm{C}$ & 0.01720 & -1.21381 & 0.48161 \\
\hline $\mathrm{C}$ & 0.00003 & 1.28313 & 0.48092 & $\mathrm{C}$ & 0.02855 & -2.45600 & -0.20831 \\
\hline $\mathrm{C}$ & 0.00005 & 1.28825 & 1.87624 & $\mathrm{H}$ & 0.01729 & -0.02376 & -1.32514 \\
\hline $\mathrm{O}$ & -0.00001 & 2.46554 & 2.61444 & $\mathrm{H}$ & -0.01737 & 3.13995 & 1.93562 \\
\hline $\mathrm{C}$ & 0.00006 & 0.08185 & 2.58230 & $\mathrm{H}$ & -0.00488 & 0.05050 & 3.64863 \\
\hline $\mathrm{C}$ & 0.00002 & -1.12252 & 1.88230 & $\mathrm{H}$ & -0.00214 & 2.14150 & -0.11563 \\
\hline $\mathrm{H}$ & -0.00002 & -2.05458 & 2.44317 & $\mathrm{~N}$ & 0.03500 & -3.47835 & -0.77176 \\
\hline $\mathrm{C}$ & 0.00002 & -1.15905 & 0.47862 & & & & \\
\hline $\mathrm{C}$ & -0.00004 & -2.47422 & -0.26426 & \multicolumn{4}{|c|}{ DimethylsulfidelRed.ISMD } \\
\hline $\mathrm{H}$ & 0.00008 & 0.07219 & -1.28999 & $\mathrm{~S}$ & -0.22511 & 0.18148 & 0.56245 \\
\hline $\mathrm{H}$ & -0.00025 & 3.21730 & 1.99536 & $\mathrm{C}$ & 0.21782 & 1.40853 & -0.71287 \\
\hline $\mathrm{H}$ & 0.00008 & 0.09507 & 3.66848 & $\mathrm{C}$ & 0.22069 & 1.11518 & 2.06502 \\
\hline
\end{tabular}




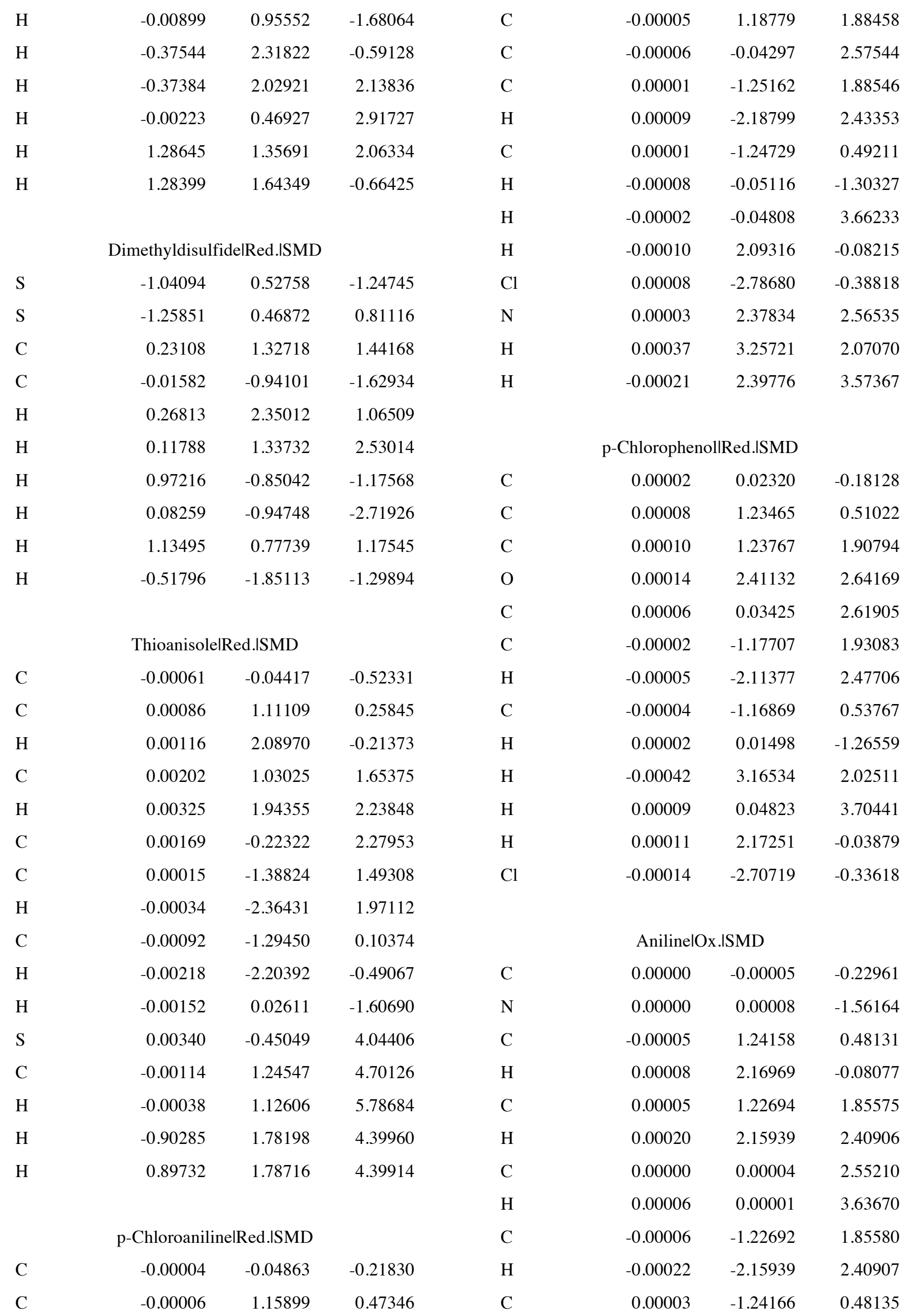




\begin{tabular}{|c|c|c|c|c|c|c|c|}
\hline $\mathrm{H}$ & -0.00009 & -2.16979 & -0.08072 & $\mathrm{H}$ & 0.87896 & -0.53225 & 4.38295 \\
\hline $\mathrm{H}$ & -0.00068 & 0.86874 & -2.08635 & $\mathrm{H}$ & -0.87811 & -0.53321 & 4.38328 \\
\hline \multirow[t]{3}{*}{$\mathrm{H}$} & 0.00079 & -0.86839 & -2.08667 & & & & \\
\hline & & & & \multicolumn{4}{|c|}{ N-MethylanilinelOx.ISMD } \\
\hline & \multicolumn{3}{|c|}{ p-MethoxyanilinelOx.ISMD } & $\mathrm{C}$ & 0.00003 & -0.05082 & -0.32836 \\
\hline $\mathrm{C}$ & 0.00003 & -0.02725 & -0.33058 & $\mathrm{C}$ & -0.00009 & 1.17788 & 0.35930 \\
\hline $\mathrm{N}$ & 0.00004 & -0.00135 & -1.66734 & $\mathrm{H}$ & -0.00019 & 2.10672 & -0.20050 \\
\hline $\mathrm{C}$ & -0.00005 & 1.19269 & 0.40956 & $\mathrm{C}$ & -0.00014 & 1.21354 & 1.73706 \\
\hline $\mathrm{H}$ & 0.00003 & 2.13511 & -0.12833 & $\mathrm{H}$ & -0.00037 & 2.16156 & 2.25984 \\
\hline $\mathrm{C}$ & 0.00002 & 1.17323 & 1.78219 & $\mathrm{C}$ & -0.00009 & -0.01415 & 2.46202 \\
\hline $\mathrm{H}$ & 0.00013 & 2.10486 & 2.33404 & $\mathrm{~N}$ & -0.00019 & -0.06439 & 3.80643 \\
\hline $\mathrm{C}$ & -0.00001 & -0.07026 & 2.46017 & $\mathrm{C}$ & 0.00019 & 1.03860 & 4.75016 \\
\hline $\mathrm{O}$ & -0.00003 & -0.19888 & 3.79706 & $\mathrm{H}$ & -0.00056 & -0.99530 & 4.21781 \\
\hline $\mathrm{C}$ & -0.00009 & -1.29094 & 1.73256 & $\mathrm{C}$ & 0.00003 & -1.25835 & 1.75757 \\
\hline $\mathrm{H}$ & -0.00028 & -2.22484 & 2.28380 & $\mathrm{H}$ & 0.00021 & -2.18174 & 2.32868 \\
\hline $\mathrm{C}$ & 0.00006 & -1.27448 & 0.36569 & $\mathrm{C}$ & 0.00011 & -1.26569 & 0.38108 \\
\hline $\mathrm{H}$ & -0.00003 & -2.19843 & -0.20310 & $\mathrm{H}$ & 0.00024 & -2.20762 & -0.15623 \\
\hline $\mathrm{H}$ & -0.00074 & 0.87614 & -2.17375 & $\mathrm{H}$ & 0.00058 & 1.99487 & 4.23625 \\
\hline $\mathrm{H}$ & 0.00075 & -0.85872 & -2.20709 & $\mathrm{H}$ & 0.88689 & 0.95439 & 5.38481 \\
\hline $\mathrm{C}$ & 0.00004 & 1.00746 & 4.61521 & $\mathrm{H}$ & -0.88667 & 0.95507 & 5.38469 \\
\hline $\mathrm{H}$ & 0.00000 & 0.64610 & 5.64132 & $\mathrm{H}$ & 0.00002 & -0.05944 & -1.41305 \\
\hline $\mathrm{H}$ & -0.90131 & 1.59107 & 4.41667 & & & & \\
\hline \multirow[t]{3}{*}{$\mathrm{H}$} & 0.90145 & 1.59097 & 4.41671 & \multicolumn{4}{|c|}{ DimethylaminelOx.ISMD } \\
\hline & & & & $\mathrm{N}$ & 0.01522 & 1.34969 & -0.70920 \\
\hline & \multicolumn{3}{|c|}{ p-MethylanilinelOx.ISMD } & $\mathrm{H}$ & 0.31492 & 2.44728 & -2.44463 \\
\hline $\mathrm{C}$ & -0.00001 & 0.00089 & -0.29993 & $\mathrm{C}$ & -0.14476 & 1.51435 & -2.12230 \\
\hline $\mathrm{N}$ & 0.00011 & -0.00360 & -1.63286 & $\mathrm{C}$ & 0.28253 & 2.42316 & 0.19969 \\
\hline $\mathrm{C}$ & -0.00004 & 1.23981 & 0.41185 & $\mathrm{H}$ & 0.26847 & 0.64634 & -2.63934 \\
\hline $\mathrm{H}$ & 0.00019 & 2.16942 & -0.14809 & $\mathrm{H}$ & 0.15992 & 3.38067 & -0.30157 \\
\hline $\mathrm{C}$ & -0.00002 & 1.23011 & 1.78496 & $\mathrm{H}$ & -1.22328 & 1.54868 & -2.34754 \\
\hline $\mathrm{H}$ & 0.00018 & 2.16882 & 2.32973 & $\mathrm{H}$ & -0.08733 & 0.41231 & -0.32162 \\
\hline $\mathrm{C}$ & -0.00013 & 0.01280 & 2.51045 & $\mathrm{H}$ & -0.37158 & 2.32881 & 1.07170 \\
\hline $\mathrm{C}$ & 0.00005 & 0.00494 & 4.00378 & $\mathrm{H}$ & 1.31629 & 2.32170 & 0.56451 \\
\hline $\mathrm{C}$ & -0.00026 & -1.21351 & 1.79136 & & & & \\
\hline $\mathrm{H}$ & -0.00050 & -2.14872 & 2.34273 & \multicolumn{4}{|c|}{ DiethylaminelOx.ISMD } \\
\hline $\mathrm{C}$ & -0.00011 & -1.23374 & 0.42115 & $\mathrm{~N}$ & -0.10104 & 0.67331 & -0.24385 \\
\hline $\mathrm{H}$ & -0.00027 & -2.16716 & -0.13244 & $\mathrm{C}$ & 0.56420 & 0.95826 & -1.48995 \\
\hline $\mathrm{H}$ & -0.00051 & 0.86282 & -2.16009 & $\mathrm{C}$ & 0.15624 & 1.38197 & 0.98440 \\
\hline $\mathrm{H}$ & 0.00084 & -0.87351 & -2.15430 & $\mathrm{C}$ & -0.18265 & 0.55971 & 2.21849 \\
\hline $\mathrm{H}$ & -0.00038 & 1.01608 & 4.41495 & $\mathrm{C}$ & -0.28689 & 0.60507 & -2.70191 \\
\hline
\end{tabular}




\begin{tabular}{|c|c|c|c|c|c|c|c|}
\hline $\mathrm{H}$ & -0.46620 & 2.29347 & 0.94683 & $\mathrm{H}$ & -5.67545 & -0.66021 & 0.17418 \\
\hline $\mathrm{H}$ & 0.88171 & 2.00457 & -1.46780 & $\mathrm{H}$ & -3.44077 & -1.85519 & -0.15113 \\
\hline $\mathrm{H}$ & -0.03647 & 1.18364 & 3.10341 & $\mathrm{H}$ & -3.51563 & -0.93759 & 1.35607 \\
\hline $\mathrm{H}$ & 0.46729 & -0.31542 & 2.29715 & $\mathrm{H}$ & -2.14951 & -0.05769 & -1.25618 \\
\hline $\mathrm{H}$ & 0.30811 & 0.76935 & -3.60342 & $\mathrm{H}$ & -1.34025 & -0.46222 & 0.26956 \\
\hline $\mathrm{H}$ & -0.58920 & -0.44606 & -2.67680 & $\mathrm{H}$ & -3.62685 & 2.58219 & -0.92744 \\
\hline $\mathrm{H}$ & -0.79530 & -0.07692 & -0.23092 & & & & \\
\hline $\mathrm{H}$ & -1.22648 & 0.23266 & 2.19809 & \multicolumn{4}{|c|}{ AnisolelOx.ISMD } \\
\hline $\mathrm{H}$ & -1.17854 & 1.23507 & -2.75126 & $\mathrm{C}$ & -0.00001 & -0.06509 & -0.41550 \\
\hline $\mathrm{H}$ & 1.19546 & 1.72445 & 0.95841 & $\mathrm{C}$ & 0.00001 & 1.16982 & 0.28328 \\
\hline $\mathrm{H}$ & 1.48856 & 0.35385 & -1.48616 & $\mathrm{H}$ & 0.00003 & 2.09787 & -0.27650 \\
\hline & & & & $\mathrm{C}$ & 0.00003 & 1.19507 & 1.65530 \\
\hline & \multicolumn{3}{|c|}{ PyrrolidinelOx.ISMD } & $\mathrm{H}$ & 0.00013 & 2.13151 & 2.19832 \\
\hline $\mathrm{C}$ & -0.24822 & -2.37882 & -0.90566 & $\mathrm{C}$ & 0.00005 & -0.04924 & 2.35191 \\
\hline $\mathrm{C}$ & -0.95534 & -2.58684 & -2.24903 & $\mathrm{C}$ & -0.00004 & -1.29940 & 1.64966 \\
\hline $\mathrm{C}$ & -0.80139 & -1.21948 & -2.94389 & $\mathrm{H}$ & -0.00014 & -2.21440 & 2.23072 \\
\hline $\mathrm{C}$ & -0.89599 & -0.22393 & -1.78283 & $\mathrm{C}$ & -0.00006 & -1.29447 & 0.27796 \\
\hline $\mathrm{N}$ & -0.40343 & -0.96867 & -0.65127 & $\mathrm{H}$ & -0.00008 & -2.22671 & -0.27437 \\
\hline $\mathrm{H}$ & -0.18570 & -0.53789 & 0.24616 & $\mathrm{H}$ & 0.00003 & -0.06028 & -1.49975 \\
\hline $\mathrm{H}$ & 0.83457 & -2.58116 & -0.96696 & $\mathrm{O}$ & 0.00021 & -0.15805 & 3.66811 \\
\hline $\mathrm{H}$ & -0.62218 & -2.95548 & -0.05469 & $\mathrm{C}$ & -0.00002 & 1.04371 & 4.50840 \\
\hline $\mathrm{H}$ & -2.01081 & -2.81459 & -2.07453 & $\mathrm{H}$ & 0.00016 & 0.66137 & 5.52527 \\
\hline $\mathrm{H}$ & -0.50960 & -3.40505 & -2.81494 & $\mathrm{H}$ & -0.90330 & 1.62014 & 4.30588 \\
\hline $\mathrm{H}$ & -1.56998 & -1.03460 & -3.69454 & $\mathrm{H}$ & 0.90290 & 1.62066 & 4.30578 \\
\hline $\mathrm{H}$ & 0.18052 & -1.13516 & -3.41825 & & & & \\
\hline $\mathrm{H}$ & -1.93819 & 0.05901 & -1.55736 & \multicolumn{4}{|c|}{ IndolelOx.ISMD } \\
\hline \multirow[t]{3}{*}{$\mathrm{H}$} & -0.33811 & 0.71137 & -1.88596 & $\mathrm{C}$ & 2.14941 & -0.66765 & 0.00000 \\
\hline & & & & $\mathrm{C}$ & 2.16510 & 0.72882 & 0.00000 \\
\hline & \multicolumn{3}{|c|}{ PiperidinelOx.ISMD } & $\mathrm{C}$ & 0.97274 & 1.45786 & 0.00000 \\
\hline $\mathrm{C}$ & -4.83330 & 1.31274 & 0.27325 & $\mathrm{C}$ & -0.24760 & 0.74909 & 0.00002 \\
\hline $\mathrm{C}$ & -4.78016 & -0.17382 & -0.22301 & $\mathrm{C}$ & -0.22672 & -0.67302 & 0.00003 \\
\hline $\mathrm{C}$ & -3.49613 & -0.84103 & 0.26397 & $\mathrm{C}$ & 0.93617 & -1.40333 & 0.00004 \\
\hline $\mathrm{C}$ & -2.25793 & -0.05898 & -0.16766 & $\mathrm{H}$ & 0.98064 & 2.54260 & 0.00000 \\
\hline $\mathrm{C}$ & -2.36218 & 1.42557 & 0.32696 & $\mathrm{H}$ & 3.08664 & -1.21371 & -0.00008 \\
\hline $\mathrm{N}$ & -3.61540 & 1.95865 & -0.12234 & $\mathrm{H}$ & 3.11487 & 1.25204 & 0.00001 \\
\hline $\mathrm{H}$ & -4.88334 & 1.30603 & 1.36654 & $\mathrm{C}$ & -1.60260 & 1.14385 & 0.00005 \\
\hline $\mathrm{H}$ & -5.68199 & 1.84736 & -0.14705 & $\mathrm{H}$ & -2.00583 & 2.14620 & 0.00001 \\
\hline $\mathrm{H}$ & -1.54828 & 2.03593 & -0.05745 & $\mathrm{C}$ & -2.37947 & -0.04984 & 0.00003 \\
\hline $\mathrm{H}$ & -2.35897 & 1.42139 & 1.42141 & $\mathrm{~N}$ & -1.56602 & -1.10358 & -0.00001 \\
\hline & -4.84017 & -0.17998 & -1.31523 & $\mathrm{H}$ & -3.45280 & -0.16433 & -0.00007 \\
\hline
\end{tabular}




\begin{tabular}{|c|c|c|c|c|c|c|c|}
\hline $\mathrm{H}$ & -1.86823 & -2.07168 & -0.00006 & \multicolumn{4}{|c|}{ PhenollOx.ISMD } \\
\hline $\mathrm{H}$ & 0.94101 & -2.48752 & 0.00010 & $\mathrm{C}$ & -0.00011 & -0.00005 & -0.24652 \\
\hline & & & & $\mathrm{H}$ & -0.00016 & -0.00004 & -1.33083 \\
\hline \multicolumn{4}{|c|}{ p-MethoxyphenollOx.ISMD } & $\mathrm{C}$ & 0.00003 & 1.23472 & 0.44818 \\
\hline $\mathrm{C}$ & 0.04825 & 0.23637 & -0.16239 & $\mathrm{H}$ & 0.00010 & 2.16303 & -0.11135 \\
\hline $\mathrm{C}$ & 0.13258 & 1.38224 & 0.68462 & $\mathrm{C}$ & 0.00008 & 1.25103 & 1.81855 \\
\hline $\mathrm{H}$ & 0.24123 & 2.35838 & 0.22617 & $\mathrm{H}$ & 0.00025 & 2.16902 & 2.39437 \\
\hline $\mathrm{C}$ & 0.07556 & 1.23010 & 2.04165 & $\mathrm{C}$ & -0.00005 & 0.00014 & 2.52449 \\
\hline $\mathrm{H}$ & 0.13728 & 2.08392 & 2.70833 & $\mathrm{O}$ & -0.00022 & 0.00015 & 3.81037 \\
\hline $\mathrm{C}$ & -0.06750 & -0.07455 & 2.58291 & $\mathrm{H}$ & -0.00008 & -0.00058 & 4.77797 \\
\hline $\mathrm{O}$ & -0.12971 & -0.29468 & 3.89983 & $\mathrm{C}$ & -0.00018 & -1.25084 & 1.81862 \\
\hline $\mathrm{H}$ & -0.06354 & 0.54799 & 4.39304 & $\mathrm{H}$ & 0.00052 & -2.16878 & 2.39448 \\
\hline $\mathrm{C}$ & -0.15216 & -1.21854 & 1.74315 & $\mathrm{C}$ & -0.00014 & -1.23474 & 0.44824 \\
\hline $\mathrm{H}$ & -0.26103 & -2.19567 & 2.19939 & $\mathrm{H}$ & 0.00004 & -2.16310 & -0.11123 \\
\hline $\mathrm{C}$ & -0.09520 & -1.06890 & 0.38312 & & & & \\
\hline $\mathrm{H}$ & -0.15880 & -1.93454 & -0.26351 & \multicolumn{4}{|c|}{ p-CyanophenollOx.ISMD } \\
\hline $\mathrm{O}$ & 0.11408 & 0.49406 & -1.46563 & $\mathrm{C}$ & 0.01235 & 0.01767 & -0.25912 \\
\hline $\mathrm{C}$ & 0.03657 & -0.61139 & -2.42043 & $\mathrm{C}$ & 0.00162 & 1.21436 & 0.40002 \\
\hline $\mathrm{H}$ & 0.11332 & -0.13602 & -3.39495 & $\mathrm{C}$ & -0.00441 & 1.21165 & 1.82921 \\
\hline $\mathrm{H}$ & 0.87218 & -1.29332 & -2.25556 & $\mathrm{O}$ & -0.01446 & 2.32901 & 2.52477 \\
\hline $\mathrm{H}$ & -0.92342 & -1.11850 & -2.31198 & $\mathrm{C}$ & 0.00008 & -0.00283 & 2.58540 \\
\hline & & & & $\mathrm{C}$ & 0.01099 & -1.19527 & 1.91845 \\
\hline \multicolumn{4}{|c|}{ p-MethylphenollOx.ISMD } & $\mathrm{H}$ & 0.01489 & -2.13645 & 2.45299 \\
\hline $\mathrm{C}$ & 0.00011 & 0.09542 & -0.23262 & $\mathrm{C}$ & 0.01715 & -1.19448 & 0.49392 \\
\hline $\mathrm{C}$ & -0.00001 & 1.28829 & 0.43993 & $\mathrm{C}$ & 0.02822 & -2.43106 & -0.19388 \\
\hline $\mathrm{C}$ & 0.00003 & 1.27125 & 1.86492 & $\mathrm{H}$ & 0.01713 & -0.02624 & -1.34072 \\
\hline $\mathrm{O}$ & 0.00003 & 2.38986 & 2.57959 & $\mathrm{H}$ & -0.01692 & 3.11779 & 1.94013 \\
\hline $\mathrm{C}$ & -0.00001 & 0.04657 & 2.59494 & $\mathrm{H}$ & -0.00525 & 0.05482 & 3.66698 \\
\hline $\mathrm{C}$ & 0.00008 & -1.13112 & 1.90191 & $\mathrm{H}$ & -0.00243 & 2.15983 & -0.13112 \\
\hline $\mathrm{H}$ & 0.00008 & -2.07587 & 2.43518 & $\mathrm{~N}$ & 0.03527 & -3.45249 & -0.75808 \\
\hline $\mathrm{C}$ & 0.00013 & -1.14311 & 0.47292 & & & & \\
\hline $\mathrm{C}$ & 0.00001 & -2.43764 & -0.25317 & \multicolumn{4}{|c|}{ DimethylsulfidelOx.ISMD } \\
\hline $\mathrm{H}$ & -0.00003 & 0.08197 & -1.31710 & $S$ & -0.21283 & 0.22140 & 0.56693 \\
\hline $\mathrm{H}$ & 0.00004 & 3.17818 & 1.99718 & $\mathrm{C}$ & 0.21454 & 1.38969 & -0.70737 \\
\hline $\mathrm{H}$ & -0.00015 & 0.08327 & 3.67818 & $\mathrm{C}$ & 0.21334 & 1.10056 & 2.05581 \\
\hline $\mathrm{H}$ & -0.00027 & 2.24075 & -0.08033 & $\mathrm{H}$ & -0.02005 & 0.92846 & -1.66531 \\
\hline $\mathrm{H}$ & 0.00005 & -2.30574 & -1.33556 & $\mathrm{H}$ & -0.36879 & 2.30275 & -0.54927 \\
\hline $\mathrm{H}$ & -0.87565 & -3.03300 & 0.04101 & $\mathrm{H}$ & -0.37455 & 2.02369 & 2.09286 \\
\hline \multirow[t]{2}{*}{$\mathrm{H}$} & 0.87543 & -3.03330 & 0.04111 & $\mathrm{H}$ & -0.01635 & 0.44874 & 2.89711 \\
\hline & & & & $\mathrm{H}$ & 1.27978 & 1.34616 & 2.01928 \\
\hline
\end{tabular}




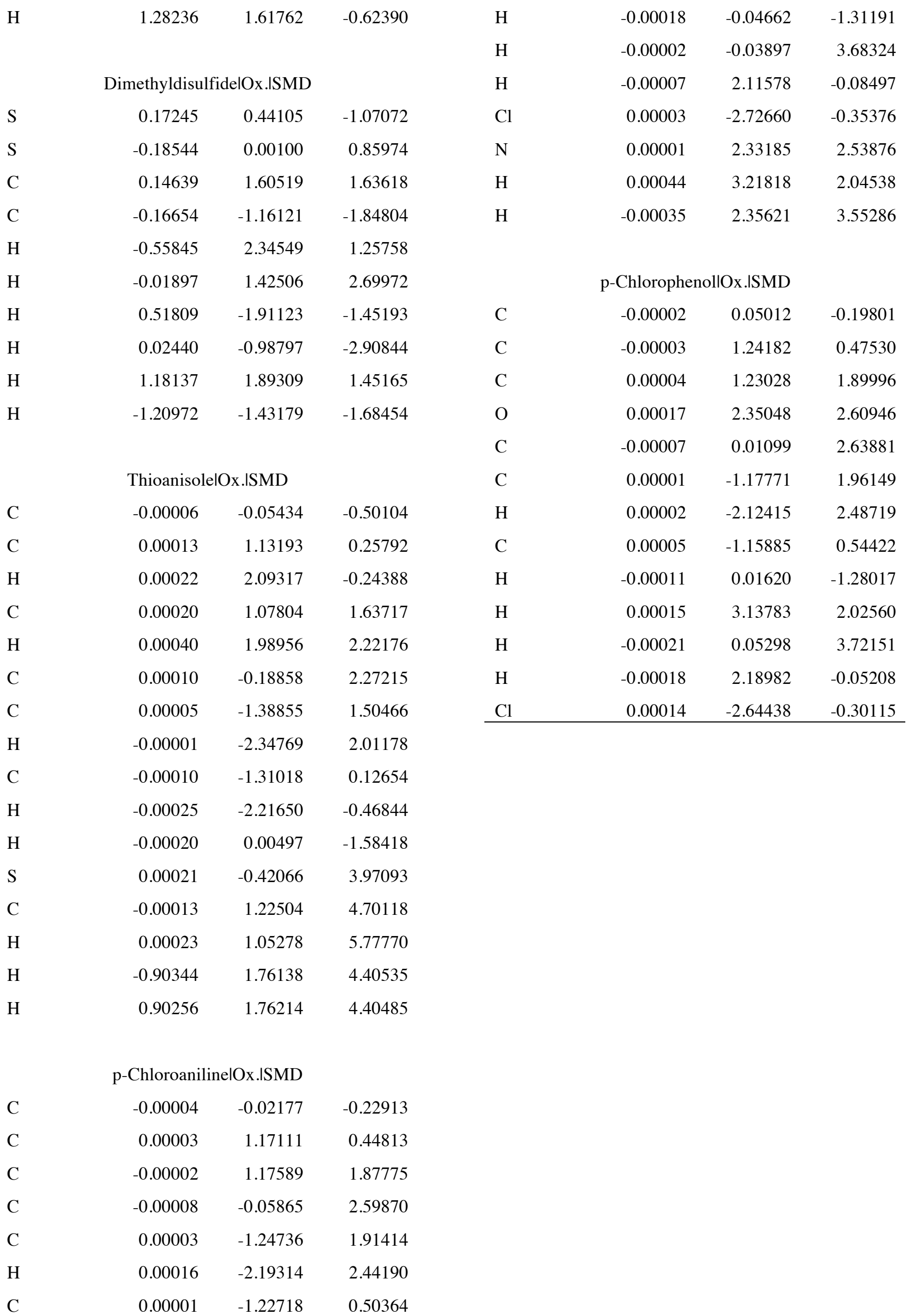

